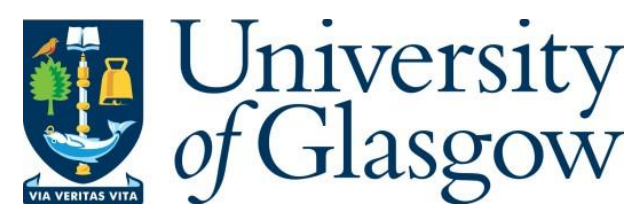

Steinmann, P., Kergaßner, A., Landkammer, P. and Zbib, H. M. (2019) A novel continuum approach to gradient plasticity based on the complementing concepts of dislocation and disequilibrium densities. Journal of the Mechanics and Physics of Solids, $132,103680$.

There may be differences between this version and the published version. You are advised to consult the publisher's version if you wish to cite from it.

$\underline{\text { http://eprints.gla.ac.uk/192740/ }}$

Deposited on: 12 September 2019

Enlighten - Research publications by members of the University of Glasgow http://eprints.gla.ac.uk 


\title{
A Novel Continuum Approach to Gradient Plasticity based on the Complementing Concepts of
}

\section{Dislocation and Disequilibrium Densities}

\author{
Paul Steinmann ${ }^{1,2, *}$, Andreas Kergaßner ${ }^{1}$, \\ Philipp Landkammer ${ }^{1}$, Hussein M. Zbib ${ }^{3}$ \\ ${ }^{1}$ Institute of Applied Mechanics, Friedrich-Alexander Universität \\ Erlangen-Nürnberg, Germany \\ ${ }^{2}$ Glasgow Computational Engineering Centre, University of Glasgow, United \\ Kingdom \\ ${ }^{3}$ School of Mechanical and Materials Engineering, Washington State \\ University, United States
}

\begin{abstract}
A geometrically linear continuum mechanics framework is proposed for gradient plasticity combining 'strain gradients' and, with a novel approach, 'stress gradients'. Thereby the duality of kinematic and kinetic quantities is exploited in view of the 'div-grad-curl orthogonality' in continuum field theories. On the one hand the non-integrability of the plastic distortion results in the well-established dislocation density - often denoted as the geometrically-necessary-dislocation (GND) density - that enters the energy storage function. On the other hand as entirely novel concept introduced in this contribution - the non-equilibrium of the plastic stress results in the disequilibrium density that parameterizes the dual dissipation potential within the convex analysis setting of plasticity. Consequently both, the dislocation density as well as the disequilibrium density contribute in modelling the size-dependent hardening state of a material in a continuum mechanics setting. The novel approach is eventually elucidated in much detail for the specific case of single crystal plasticity.
\end{abstract}

\section{Introduction}

Microscopically, the underlying cause for the plastic behaviour of metals is the emergence (e.g. from Frank-Read sources), motion (e.g. by Peach-Koehler force driven glide), interaction and arrest (e.g. at pileups) of discrete dislocations. Thereby the spacing between two discrete dislocations is typically in the order of only a few lattice spacings (some few nm), making it prohibitive to simulate material samples of meso- and macroscopic dimensions, for example by Molecular Dynamics (MD), see e.g. Tschopp, Spearot and McDowell [58], Bitzek and Gumbsch [7, 8] for relevant MD system sizes, or Discrete Dislocation Dynamics (DDD), see e.g. Hirth, Rhee and Zbib [34], Zbib, Rhee and Hirth [66], Zbib et 
al. [65], Zbib, De la Rubia and Bulatov [64] for relevant DDD system sizes.

From a continuum perspective, plasticity is modelled at the macro scale in terms of continuously distributed external and especially internal (field) variables such as for example the plastic distortion - that capture the collective or rather homogenized effects of the discretely, and from a macroscopic viewpoint densely distributed dislocations at the micro scale. Classical continuum plasticity, however, is void of any internal length scale, making it thus impossible to account for the size-dependent hardening behaviour of metals consistently observed in numerous experiments at the meso scale, see e.g. Fleck et al. [16], Stölken and Evans [56], Uchic et al. [59], Ehrler et al. [13], Dunstan et al. [12], Liu et al. $[41,40]$. As a remedy, it has been proposed to firstly model the distribution of dislocations at the meso scale in terms of the continuum dislocation density that essentially decomposes into the Statistically Stored Dislocation (SSD) and the Geometrically Necessary Dislocation (GND) density, whereby the concept of the continuum dislocation density goes back to the early works by Nye [49], Bilby, Bullough and Smith [6], Kröner [37, 35], Kröner and Seeger [36]. An overview on the foundations of continuum defect densities in differential geometry is given in Steinmann [55].

Then, on the one hand, 'strain gradient' formulations of plasticity have been advocated by incorporating in one way or other the continuum dislocation density into the modelling, a non-comprehensive selection of the countless contributions to 'strain gradient' plasticity is given by the contributions of Zbib and Aifantis [61, 62, 63], Steinmann [54], Fleck and Hutchinson [15], Menzel and Steinmann [48], Gurtin [21, 22, 23, 24, 25], Gudmundson [20], Gurtin and Anand [26, 27, 29, 28], Evans and Hutchinson [14], Forest [17], Forest and Aifantis [18], Reddy [52, 53], Gurtin and Reddy [30], McBride, Reddy and Steinmann [46], and many others.

On the other hand, as a complimentary approach and motivated by the classical Hall-Petch inverse relation between the yield resistance and the square root of the grain size (Hall [31], Petch [50]), plasticity models incorporating 'stress gradients' have been advocated by considering the size-dependent behaviour resulting from pileup of dislocations at obstacles, whereby a representative selection of the comparatively few contributions to 'stress gradient' plasticity is given by the works of Hirth [33], Chakravarthy and Curtin [10], Akarapu and Hirth [1], Liu, He and Zhang [42], Liu et al. [43], Taheri-Nassaj and Zbib [57]. Here, typically, the gradient of the applied stress that enters the equilibrium equation, i.e. the stress vanishing for zero external loads (except the residual stress that is in self-equilibrium), contributes to the yield resistance, thus making it size-dependent.

Combined 'strain' and 'stress gradient' plasticity has been successfully applied to capture, e.g., the multi-scale nature of size-dependence in heterogeneous nanoand micro-structured materials, see Lyu, Taheri-Nassay and Zbib [45], Lyu et 
al. [44].

In stark contrast to the established genuine formulations of 'stress gradient' plasticity in the above that are motivated by considering dislocation pileup problems (and similarly to the 'stress gradient' elasticity approaches by Forest and Sab [19] and Polizotto [51] that require additional degrees of freedom that expand work with the stress gradients), our novel approach towards gradient plasticity combining 'strain' and - especially - 'stress gradients' takes however a purely continuum mechanics view and thus rests on the following philosophy:

Two conditions of complementing flavour always need to be satisfied identically in continuum mechanics. These are integrability of the distortion $\boldsymbol{\beta}$ and equilibrium of the stress $\sigma$, which are expressed (for convenience of exposition but without lack of generality for the case of vanishing body force density) as

$$
\operatorname{curl} \boldsymbol{\beta} \doteq \mathbf{0} \quad \text { and } \quad \operatorname{div} \boldsymbol{\sigma} \doteq \mathbf{0} .
$$

Guided by these complementing requirements we present an argument that is based on the duality of kinematic and kinetic quantities: on the one hand we consider the lack of integrability (i.e. non-integrabibility) of kinematic quantities, i.e. the plastic distortion $\boldsymbol{\beta}_{\mathrm{p}}$, and on the other hand we consider the lack of equilibrium (i.e. non-equilibrium) of kinetic quantities, i.e. the here proposed concept of plastic stress $\sigma_{\mathrm{p}}$. Then, on the one hand, this duality argument is used to motivate the consideration of the dislocation density $\boldsymbol{\alpha}_{\mathrm{p}}$ as 'strain gradient' contribution in the energy storage function $W$. Likewise, on the other hand, it motivates the here newly introduced disequilibrium density $\varrho_{\mathrm{p}}$ as 'stress gradient' contribution in the dual dissipation potential $D^{*}$. Thereby, the convex but possibly non-smooth dual dissipation potential essentially encapsulates the yield condition and the flow rule of plasticity (for an exposition of the details that follows the convex analysis setting by Han and Reddy [32] see below).

The manuscript is structured as follows: Section 2 introduces the complementing key concepts, i.e. 2.1) the plastic distortion and the plastic stress, 2.2) the energy storage function and the dissipation potential for local plasticity, the incorporation of 2.3) the dislocation density (representing the non-integrability of the plastic distortion) for 'strain gradient' plasticity, and 2.4) the novel concept of the disequilibrium density (representing the non-equilibrium of the plastic stress) for 'stress gradient' plasticity, all for the case of the phenomenological setting of plasticity. Section 3 then applies these generic concepts to the specific case of single crystal plasticity, by 3.1) reviewing the formulation of rate-independent local single crystal plasticity, expressing the single crystal specific 3.2) dislocation density and 3.3) disequilibrium density, both expressed in terms of the individual slip system's plastic slip gradients, 3.4) illustrating these concepts for the analytical example of edge dislocation induced bending, and eventually 3.5) extending the local formulation to gradient single crystal plasticity combining 'strain gradients' and 'stress gradients'. Section 4 focuses 
computationally on the size-dependent hardening resulting from the consideration of dislocation and disequilibrium densities in the torsion problem of a single crystalline micro wire. Finally Section 5 concludes our findings.

\section{Motivation: Phenomenological Plasticity}

To set the stage, relevant concepts and formulations are first introduced within the geometrically linear setting of phenomenological plasticity. Thereby traditional local plasticity is extended to gradient plasticity combining 'strain gradients' and 'stress gradients' by the incorporation of the dislocation density and the novel concept of disequilibrium density, respectively.

\subsection{Plastic Distortion and Plastic Stress}
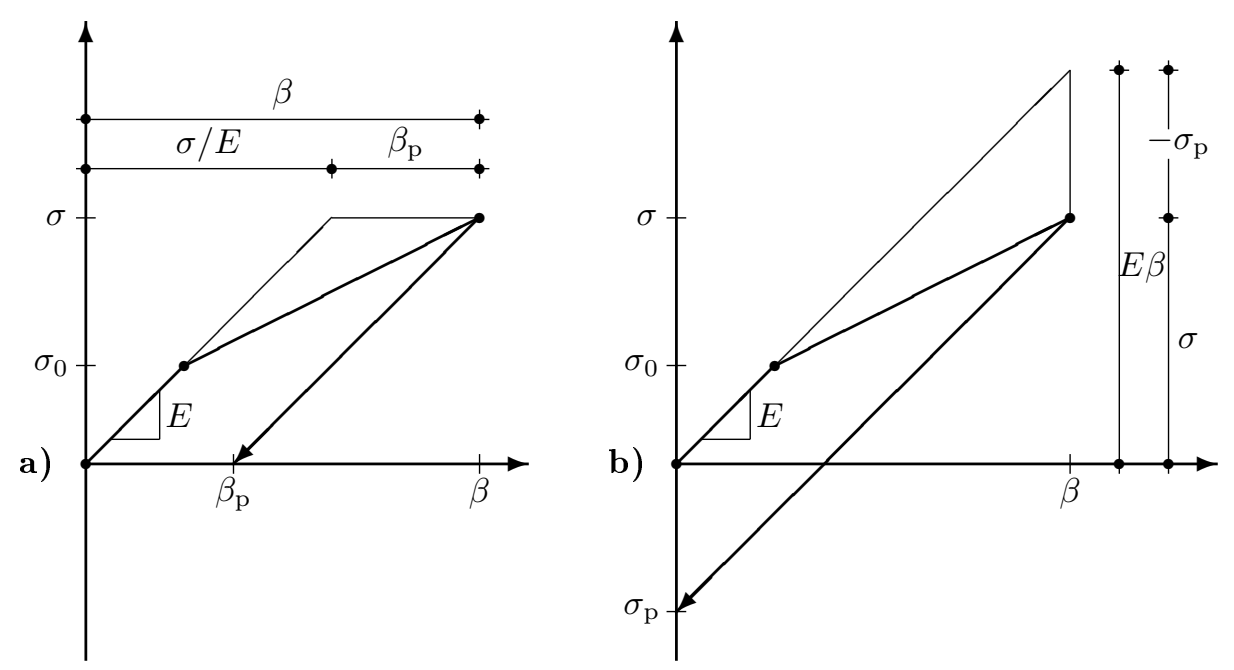

Figure 1: Operational definition of plastic distortion $\beta_{\mathrm{p}}$ and plastic stress $\sigma_{\mathrm{p}}$ for (without loss of generality) the case of one-dimensional linear elastoplasticity with elasticity modulus $E$, yield stress $\sigma_{0}$, and linear hardening. A one-dimensional sample is uniaxially loaded beyond the yield limit $\sigma_{0}$ up to a state with stress $\sigma$ and distortion $\beta$ in the elasto-plastic regime, the corresponding bilinear stress-distortion diagram is depicted by the bold lines. For subsequent stress-driven unloading with $\sigma \rightarrow 0$, as displayed in a) by the bold arrow, the plastic distortion is identified as the remaining distortion $\beta_{\mathrm{p}}$. The decomposition $\beta=\sigma / E+\beta_{\mathrm{p}}$ is clearly recognized from the diagram. Vice versa for distortion-driven unloading with $\beta \rightarrow 0$, as displayed in $\mathbf{b}$ ) by the bold arrow, the plastic stress is identified as the remaining (negative) stress $\sigma_{\mathrm{p}}$. The decomposition $\sigma=E \beta+\sigma_{\mathrm{p}}$ is clearly recognized from the diagram. 
In geometrically linear continuum mechanics the key kinematic quantity is the displacement field $\boldsymbol{u}=\boldsymbol{u}(\boldsymbol{x})$ that relates positions $\boldsymbol{x} \in \mathcal{B}$ in the (initial) configuration $\mathcal{B} \subset \mathbb{E}^{3}$ to their displacements $\boldsymbol{u} \in \mathbb{R}^{3}$. Then the total distortion $\boldsymbol{\beta}$ is defined as gradient of the displacement field $\boldsymbol{u}$, consequently it is integrable as expressed by the curl operator curl $\{\bullet\}$

$$
\boldsymbol{\beta}:=\operatorname{grad} \boldsymbol{u} \quad \text { with } \quad \operatorname{curl} \boldsymbol{\beta}:=-\beta_{i j, k} e_{j k l} \boldsymbol{e}_{i} \otimes \boldsymbol{e}_{l} \doteq \mathbf{0} .
$$

Here $e_{j k l}$ denotes the coefficients of the permutation symbol and $\boldsymbol{e}_{i}$ are the Cartesian base vectors. Consequently, the coefficients of curl $\boldsymbol{\beta}$ expand for later use as

$$
[\operatorname{curl} \boldsymbol{\beta}]_{i j}=\left[\begin{array}{ccc}
\beta_{13,2}-\beta_{12,3} & \beta_{11,3}-\beta_{13,1} & \beta_{12,1}-\beta_{11,2} \\
\beta_{23,2}-\beta_{22,3} & \beta_{21,3}-\beta_{23,1} & \beta_{22,1}-\beta_{21,2} \\
\beta_{33,2}-\beta_{32,3} & \beta_{31,3}-\beta_{33,1} & \beta_{32,1}-\beta_{31,2}
\end{array}\right]
$$

Remark: As an aside, the total strain $\boldsymbol{\epsilon}:=\boldsymbol{\beta}^{\mathrm{sym}}=\operatorname{grad}^{\mathrm{sym}} \boldsymbol{u}$ follows as the symmetric gradient of the displacement field, consequently it satisfies the St. Venant compatibility conditions inc $\boldsymbol{\epsilon}:=e_{m i j} \epsilon_{i k, j l} e_{k l n} \boldsymbol{e}_{m} \otimes \boldsymbol{e}_{n} \doteq \mathbf{0}$ as expressed by the Kröner incompatibility operator inc $\{\bullet\}$, refer to Steinmann [55].

The following concepts of plastic distortion and plastic stress are schematically depicted in Fig. 1.

For geometrically linear (local) phenomenological plasticity the applied stress $\boldsymbol{\sigma}$ - satisfying equilibrium - depends generically on the (symmetric parts of the) total distortion $\boldsymbol{\beta}$ and the plastic distortion $\boldsymbol{\beta}_{\mathrm{p}}$ that serves as internal variable

$$
\boldsymbol{\sigma}=\boldsymbol{\sigma}\left(\boldsymbol{\beta}^{\mathrm{sym}}, \boldsymbol{\beta}_{\mathrm{p}}^{\mathrm{sym}}\right) \quad \text { with } \quad \operatorname{div} \boldsymbol{\sigma}:=\sigma_{i j, j} \boldsymbol{e}_{i} \doteq \mathbf{0} .
$$

Thereby the (symmetric part of the) plastic distortion $\boldsymbol{\beta}_{\mathrm{p}}^{\mathrm{sym}}$ is in general defined as the (symmetric part of the) distortion $\boldsymbol{\beta}^{\mathrm{sym}}$ at locally vanishing stress $\boldsymbol{\sigma} \equiv \mathbf{0}$, i.e. as the (symmetric part of the) in general non-integrable distortion $\boldsymbol{\beta}_{\mathrm{p}}$ that remains after stress-driven point-wise (elastic) unloading

$$
\left.\boldsymbol{\beta}_{\mathrm{p}}^{\text {sym }} \stackrel{\text { locally }}{:=} \boldsymbol{\beta}^{\text {sym }}\right|_{\sigma \equiv 0} \quad \text { with } \quad \operatorname{curl} \boldsymbol{\beta}_{\mathrm{p}} \neq \mathbf{0} \quad \Longrightarrow \quad \text { inc } \boldsymbol{\beta}_{\mathrm{p}}^{\mathrm{sym}} \neq \mathbf{0} .
$$

From the above generic constitutive relation for $\sigma$, the (symmetric part of the) distortion at locally vanishing stress computes concretely as

$$
\left.\boldsymbol{\beta}^{\mathrm{sym}}\right|_{\sigma \equiv 0}=\arg \left\{\left.\boldsymbol{\sigma}\left(\boldsymbol{\beta}^{\mathrm{sym}}, \boldsymbol{\beta}_{\mathrm{p}}^{\mathrm{sym}}\right)\right|_{\beta_{\mathrm{p}}}=\mathbf{0}\right\} .
$$


Remark: Note that in (local) phenomenological plasticity it is indeed only the symmetric part $\boldsymbol{\beta}_{\mathrm{p}}^{\mathrm{sym}}$ of the plastic distortion rather than the plastic distortion $\boldsymbol{\beta}_{\mathrm{p}}$ that enters the constitutive modelling, with the skew-symmetric part $\boldsymbol{\beta}_{\mathrm{p}}^{\text {skw }}$ being undetermined. Thus, basically only the incompatibility inc $\beta_{\mathrm{p}}^{\text {sym }}$ rather than the non-integrability curl $\boldsymbol{\beta}_{\mathrm{p}}$ can practically be determined in phenomenological plasticity. However, although it is possible to derive a phenomenological 'strain gradient' plasticity based on the consideration of inc $\boldsymbol{\beta}_{\mathrm{p}}^{\mathrm{sym}}$ (see Menzel and Steinmann [48]), in order to avoid the technicalities stemming from the second gradients contained in inc $\boldsymbol{\beta}_{\mathrm{p}}^{\mathrm{sym}}$ and since our main objective is to eventually consider single crystal plasticity, in which $\boldsymbol{\beta}_{\mathrm{p}}$ is fully given constitutively, we base our motivation in phenomenological plasticity for the sake of argument also on the plastic distortion (being fully aware that inc $\boldsymbol{\beta}_{\mathrm{p}}^{\mathrm{sym}}$ is more challenging to evaluate and to process than curl $\boldsymbol{\beta}_{\mathrm{p}}$, for an in-depth discussion see [48]).
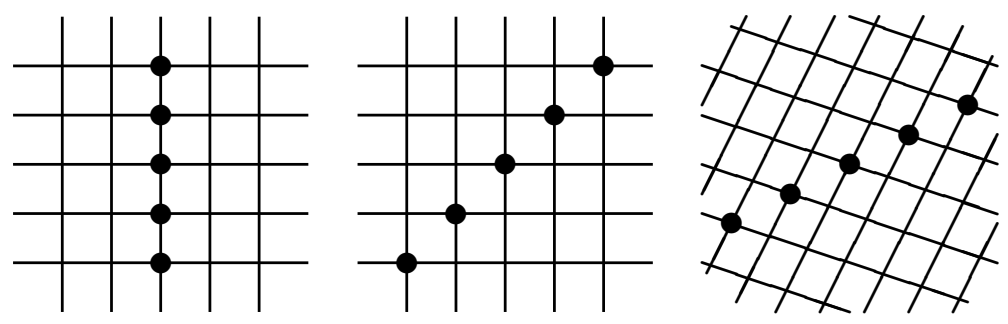

Figure 2: Additive decomposition of the distortion $\boldsymbol{\beta}$ in an idealized crystalline lattice into plastic and elastic contributions $\boldsymbol{\beta}_{\mathrm{p}}$ and $\boldsymbol{\beta}_{\mathrm{e}}$, respectively. Due to the dislocation flow along the slip directions the plastic distortion $\boldsymbol{\beta}_{\mathrm{p}}$ leaves the crystalline lattice unaffected (middle), the elastic distortion $\boldsymbol{\beta}_{\mathrm{e}}$, however, distorts the lattice (right) and is thus responsible for the generation of stress.

Typically, see Fig. 2, the distortion $\boldsymbol{\beta}$ is considered to decompose additively into the elastic (i.e. stress producing) distortion $\boldsymbol{\beta}_{\mathrm{e}}$ and the plastic (i.e. lattice preserving) distortion $\boldsymbol{\beta}_{\mathrm{p}}$, i.e.

$$
\boldsymbol{\beta}=\boldsymbol{\beta}_{\mathrm{e}}+\boldsymbol{\beta}_{\mathrm{p}}
$$

Here it is crucial to observe that whereas $\boldsymbol{\beta}=\operatorname{grad} \boldsymbol{u}$ is clearly integrable, the plastic distortion $\boldsymbol{\beta}_{\mathrm{p}}$ and likewise the elastic distortion $\boldsymbol{\beta}_{\mathrm{e}}$ are in general non-integrable. Physically, the plastic distortion $\boldsymbol{\beta}_{\mathrm{p}}$ is a measure for the local, irreversible and non-integrable deformation $\boldsymbol{\beta}_{\mathrm{p}} \cdot \mathrm{d} \boldsymbol{x}$ that results from all dislocations that have already left the local neighbourhood of a material point $\boldsymbol{x}$, thereby leaving the crystal lattice unaffected. For example, under the action of the plastic distortion, an initial cube with $C^{0}$-continuous, smooth almost everywhere boundary transforms into a parallelepiped with $C^{-1}$-(dis)continuous 
(staircase-like) boundary, see Fig. 2. The evolution of the plastic distortion $\boldsymbol{\beta}_{\mathrm{p}}$ is thus associated with the flow of dislocations through the crystal lattice, i.e. with the rate at which dislocations are entering and/or leaving the local neighbourhood of a material point $\boldsymbol{x}$.

Then, based on the additive decomposition, and for the sake of concreteness, the constitutive relation for $\boldsymbol{\sigma}$ in the case of geometrically linear, phenomenological plasticity is explicitly given as

$$
\boldsymbol{\sigma}=\boldsymbol{E}:\left[\boldsymbol{\beta}-\boldsymbol{\beta}_{\mathrm{p}}\right]^{\mathrm{sym}} \equiv \boldsymbol{E}:\left[\boldsymbol{\beta}-\boldsymbol{\beta}_{\mathrm{p}}\right] .
$$

Here $\boldsymbol{E}$ denotes the fourth-order, possibly anisotropic, elasticity tensor, obeying minor and major symmetries, thus allowing to omit symmetrization of the (total) distortion and the plastic distortion in the above constitutive expression. For elastic isotropy $\boldsymbol{E}$ expands as $\boldsymbol{E}=2 G \boldsymbol{I}^{\text {sym }}+L \mathbf{1} \otimes \mathbf{1}$ where $G$ and $L$ are the classical Lamé constants and $\boldsymbol{I}^{\mathrm{sym}}$ and $\mathbf{1}$ denote the (symmetric) fourth- and second-order unit tensors, respectively.
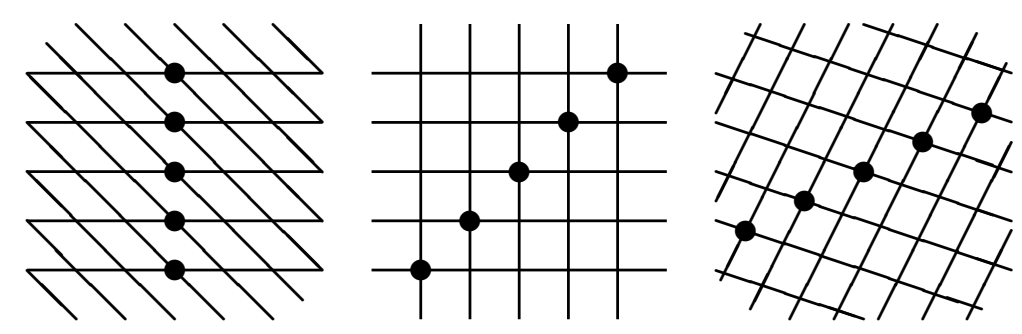

Figure 3: Local distortion-driven unloading to $\boldsymbol{\beta}=\mathbf{0}$ and thus $\boldsymbol{\beta}_{\mathrm{e}}=-\boldsymbol{\beta}_{\mathrm{p}}$. The plastic stress $\sigma_{\mathrm{p}}$ is produced by distorting the (dislocated) crystal lattice from its non-integrable plastic configuration (middle) back into the integrable (initial) configuration $\mathcal{B}$ (left). This is in analogy to the distortion of the (dislocated) crystal lattice from its non-integrable plastic configuration (middle) into the integrable (deformed) configuration $\mathcal{B}$ (right) that produces the stress $\boldsymbol{\sigma}$ entering the equilibrium condition.

Vice versa, and as a novel concept, the plastic stress $\sigma_{\mathrm{p}}$ is defined as the stress $\boldsymbol{\sigma}$ at locally vanishing distortion $\boldsymbol{\beta} \equiv \mathbf{0}$ (which can indeed be controlled), see Fig. 3 i.e. as the non-equilibrium stress $\sigma_{\mathrm{p}}$ that arises after distortion-driven point-wise (elastic) unloading

$$
\left.\boldsymbol{\sigma}_{\mathrm{p}} \stackrel{\text { locally }}{:=} \boldsymbol{\sigma}\right|_{\beta \equiv 0} \quad \text { with } \quad \operatorname{div} \boldsymbol{\sigma}_{\mathrm{p}} \neq \mathbf{0} .
$$

Thereby the stress at locally vanishing (symmetric part of the) distortion computes concretely as 


$$
\left.\boldsymbol{\sigma}\right|_{\beta \equiv 0}=\left.\boldsymbol{\sigma}\left(\boldsymbol{\beta}^{\mathrm{sym}}, \boldsymbol{\beta}_{\mathrm{p}}^{\mathrm{sym}}\right)\right|_{\beta=0, \beta_{\mathrm{p}}}
$$

and thus the plastic stress $\sigma_{\mathrm{p}}$ expands here, based on the above specific constitutive relation for $\sigma$, as

$$
\boldsymbol{\sigma}_{\mathrm{p}}:=-\boldsymbol{E}: \boldsymbol{\beta}_{\mathrm{p}}^{\mathrm{sym}} \equiv-\boldsymbol{E}: \boldsymbol{\beta}_{\mathrm{p}} .
$$

Thus, the plastic stress relates to the (symmetric part of the) negative plastic distortion in the metric given by the elasticity tensor that captures the elastic interactions within the crystalline lattice. In other words, the plastic stress is the stress needed to elastically (i.e. at fixed plastic distortion) return from the nonintegrable plastic configuration to the integrable initial configuration (modulo infinitesimal plastic rotations described by $\boldsymbol{\beta}^{\text {skw }}$ ). Sloppily speaking, the plastic stress thus restores the initial configuration, however, while preserving the plastic distortion. It is interesting to note that the so defined plastic stress is conceptually different from - but formally related to - the stress polarisation as introduced in the seminal approach to homogenisation theory by Willis $[60]^{1}$.

\subsection{Energy Storage Function and Dissipation Potential}

For plastic solids and in the 'non-gradient' local case, the constitutive relation for the stress $\boldsymbol{\sigma}$ and the 'driving force' $\boldsymbol{\varsigma}_{\mathrm{p}}$ derive as partial derivatives $\partial(\bullet)$ from a (properly convex) energy storage function $W$ that is parameterized in the (symmetric parts of the) total distortion $\boldsymbol{\beta}$ (as the external variable) and the plastic distortion $\boldsymbol{\beta}_{\mathrm{p}}$ (as the internal variable), i.e.

$$
W=W\left(\boldsymbol{\beta}^{\mathrm{sym}}, \boldsymbol{\beta}_{\mathrm{p}}^{\mathrm{sym}}\right) \quad \text { with } \quad \boldsymbol{\sigma}=\partial_{\beta} W \quad \text { and } \quad \boldsymbol{\varsigma}_{\mathrm{p}}:=-\partial_{\beta_{\mathrm{p}}} W .
$$

Consequently, the (symmetric) stress $\boldsymbol{\sigma}$ entering the equilibrium condition and the (symmetric) 'driving force' $\varsigma_{\mathrm{p}}$ driving the evolution of the (symmetric part of the) plastic distortion $\boldsymbol{\beta}_{\mathrm{p}}$ also depend on the (symmetric parts of the) distortion $\boldsymbol{\beta}$ and the plastic distortion $\boldsymbol{\beta}_{\mathrm{p}}$

\footnotetext{
${ }^{1}$ In order to demonstrate that the concept of plastic stress is conceptually different from but formally related to - the stress polarisation as introduced in Willis' seminal homogenisation theory, the starting steps to the latter theory are briefly re-iterated. Within a composite the stress polarisation $\boldsymbol{\tau}(\boldsymbol{x})=\boldsymbol{\sigma}(\boldsymbol{x})-\boldsymbol{E}_{0}: \boldsymbol{\epsilon}(\boldsymbol{x})$ denotes the difference between the (fluctuating) stress $\boldsymbol{\sigma}(\boldsymbol{x})=\boldsymbol{E}(\boldsymbol{x}):\left[\boldsymbol{\epsilon}(\boldsymbol{x})-\boldsymbol{\epsilon}_{\mathrm{p}}(\boldsymbol{x})\right]$ in a heterogeneous material with non-constant stiffness $\boldsymbol{E}(\boldsymbol{x})$ and the stress $\boldsymbol{E}_{0}: \boldsymbol{\epsilon}(\boldsymbol{x})$ in a homogeneous comparison material with constant stiffness $\boldsymbol{E}_{0}$. Here, $\boldsymbol{\epsilon}(\boldsymbol{x})$ is the total strain in the composite compatible with the displacement field $\boldsymbol{u}(\boldsymbol{x})$ and $\epsilon_{\mathrm{p}}(\boldsymbol{x})$ is the plastic strain in the composite that, for the sake of presentation, shall serve as the only contribution to the eigenstrain. Thus, the stress polarisation may be expanded as $\boldsymbol{\tau}(\boldsymbol{x})=\left[\boldsymbol{E}(\boldsymbol{x})-\boldsymbol{E}_{0}\right]: \boldsymbol{\epsilon}(\boldsymbol{x})-\boldsymbol{E}(\boldsymbol{x}): \boldsymbol{\epsilon}_{\mathrm{p}}(\boldsymbol{x})$. The stress $\boldsymbol{\sigma}(\boldsymbol{x})$ enters the equilibrium condition $\operatorname{div} \boldsymbol{\sigma}(\boldsymbol{x})=\mathbf{0}$ that may be re-expressed in terms of the homogeneous comparison material as $\operatorname{div}\left(\boldsymbol{E}_{0}: \boldsymbol{\epsilon}(\boldsymbol{x})\right)+\operatorname{div} \boldsymbol{\tau}(\boldsymbol{x})=\mathbf{0}$. The term div $\boldsymbol{\tau}(\boldsymbol{x})$ may then be interpreted as a body force, which, when known, allows solution for $\boldsymbol{\epsilon}(\boldsymbol{x})$ in terms of the infinite-body Green's function that is known for the homogeneous comparison material. When comparing any of the above stress expressions with the here advocated plastic stress $\boldsymbol{\sigma}_{\mathrm{p}}(\boldsymbol{x}):=-\boldsymbol{E}(\boldsymbol{x}): \boldsymbol{\epsilon}_{\mathrm{p}}(\boldsymbol{x})$ (arising as the stress after a strain-driven unloading with $\boldsymbol{\epsilon}(\boldsymbol{x}) \rightarrow \mathbf{0})$, it becomes obvious that the plastic stress is indeed conceptually different from - but formally related to - the stress polarisation. Indeed, $\boldsymbol{\sigma}_{\mathrm{p}}(\boldsymbol{x})$ and $\boldsymbol{\tau}(\boldsymbol{x})$ do formally coincide for homogeneous material with $\boldsymbol{E}(\boldsymbol{x}) \equiv \boldsymbol{E}_{0}$.
} 


$$
\boldsymbol{\sigma}=\boldsymbol{\sigma}\left(\boldsymbol{\beta}^{\mathrm{sym}}, \boldsymbol{\beta}_{\mathrm{p}}^{\mathrm{sym}}\right) \quad \text { and } \quad \varsigma_{\mathrm{p}}=\varsigma_{\mathrm{p}}\left(\boldsymbol{\beta}^{\mathrm{sym}}, \boldsymbol{\beta}_{\mathrm{p}}^{\mathrm{sym}}\right) .
$$

Example: A typical constitutive choice for the energy storage function $W$ is the quadratic Hooke type model

$$
W=W\left(\boldsymbol{\beta}^{\mathrm{sym}}, \boldsymbol{\beta}_{\mathrm{p}}^{\mathrm{sym}}\right)=W\left(\left[\boldsymbol{\beta}-\boldsymbol{\beta}_{\mathrm{p}}\right]^{\mathrm{sym}}\right)=\frac{1}{2}\left[\boldsymbol{\beta}-\boldsymbol{\beta}_{\mathrm{p}}\right]: \boldsymbol{E}:\left[\boldsymbol{\beta}-\boldsymbol{\beta}_{\mathrm{p}}\right] .
$$

Then the stress $\boldsymbol{\sigma}$ computes as in Eq. 8. Likewise, the 'driving force' $\varsigma_{\mathrm{p}}$ computes as

$$
\boldsymbol{\varsigma}_{\mathrm{p}}\left(\boldsymbol{\beta}^{\mathrm{sym}}, \boldsymbol{\beta}_{\mathrm{p}}^{\text {sym }}\right)=\boldsymbol{E}:\left[\boldsymbol{\beta}-\boldsymbol{\beta}_{\mathrm{p}}\right]^{\text {sym }} \equiv \boldsymbol{E}:\left[\boldsymbol{\beta}-\boldsymbol{\beta}_{\mathrm{p}}\right]
$$

and thus coincides here identically $\left(\sigma \equiv \varsigma_{\mathrm{p}}\right)$ with the stress entering the equilibrium condition $\operatorname{div} \boldsymbol{\sigma}=\mathbf{0}$.

Within the modelling framework of (generalized) standard materials the rate of the (symmetric part of the) plastic distortion $\boldsymbol{\beta}_{\mathrm{p}}^{\mathrm{sym}}$ lies in the sub-differential $\mathfrak{d}(\bullet)$ (i.e. the set of sub-derivatives) of the convex but possibly non-smooth dual dissipation potential $D^{*}$ (indeed, for the case of rate-independent plasticity $D^{*}$ is non-smooth), i.e.

$$
D^{*}=D^{*}\left(\varsigma_{\mathrm{p}}\right) \quad \text { with } \quad \dot{\boldsymbol{\beta}}_{\mathrm{p}}^{\text {sym }} \in \mathfrak{d}_{\varsigma_{\mathrm{p}}} D^{*} .
$$

The (dual) dissipation potential needs to satisfy a number of requirements (convexity, positive homogeneity, zero at the origin) for the modelling to be thermodynamically or rather dissipation consistent (i.e. automatically satisfying the requirement of the second law of thermodynamics for positive dissipation). For rate-independent plasticity the convex but non-smooth (dual) dissipation potential $D^{*}$ is the indicator function I of the admissible domain

$$
D^{*}=I\left(Y\left(\varsigma_{\mathrm{p}}\right)\right) \quad \text { with } \quad I(Y):=\left\{\begin{array}{ccc}
0 & & Y \leq 0 \\
& \text { for } & \\
\infty & & \text { else }
\end{array}\right.
$$

that is expressed in terms of a convex yield condition

$$
Y\left(\varsigma_{\mathrm{p}}\right) \leq 0
$$

Then the evolution of the (symmetric part of the) plastic distortion $\boldsymbol{\beta}_{\mathrm{p}}^{\text {sym }}$ follows alternatively as

$$
\dot{\boldsymbol{\beta}}_{\mathrm{p}}^{\text {sym }}=\lambda \partial_{\varsigma_{\mathrm{p}}} Y,
$$

whereby $\lambda \geq 0$ denotes a positive multiplier that follows from the Karush-KuhnTucker complementary conditions together with the consistency condition

$$
Y \leq 0, \quad \lambda \geq 0, \quad \lambda Y=0, \quad \text { and } \quad \lambda \dot{Y}=0 .
$$


In phenomenological plasticity the Karush-Kuhn-Tucker complementary conditions are also denoted the loading-unloading conditions, with $\lambda$ the plastic multiplier following from the consistency condition.

Example: For phenomenological (poly-crystalline) metal plasticity a typical constitutive choice for the yield condition $Y$ is the v. Mises model

$$
Y=Y\left(\boldsymbol{\varsigma}_{\mathrm{p}}\right)=\left|\boldsymbol{\varsigma}_{\mathrm{p}}^{\mathrm{dev}}\right|-\tau_{0} \leq 0 .
$$

Here $\varsigma_{\mathrm{p}}^{\mathrm{dev}}:=\boldsymbol{I}^{\mathrm{dev}}: \boldsymbol{}_{\mathrm{p}}$ denotes the deviatoric part of the 'driving force' with $\boldsymbol{I}^{\mathrm{dev}}:=\boldsymbol{I}^{\mathrm{sym}}-\frac{1}{3} \mathbf{1} \otimes \mathbf{1}$ the symmetric fourth-order deviatoric projection operator, and $\tau_{0}:=\sqrt{2 / 3} \sigma_{0}$ is a properly defined yield limit in terms of the uniaxial yield stress $\sigma_{0}$. The corresponding (associated) evolution law for the (symmetric part of the) plastic distortion then follows as

$$
\dot{\boldsymbol{\beta}}_{\mathrm{p}}^{\mathrm{sym}}=\lambda \frac{\boldsymbol{\varsigma}_{\mathrm{p}}^{\text {dev }}}{\left|\boldsymbol{\boldsymbol { \rho }}_{\mathrm{p}}^{\text {dev }}\right|} \quad \text { with } \quad \lambda=\left|\dot{\boldsymbol{\beta}}_{\mathrm{p}}^{\mathrm{sym}}\right| \quad \text { and } \quad\left|\boldsymbol{\varsigma}_{\mathrm{p}}^{\text {dev }}\right|=\tau_{0} .
$$

Observe that in phenomenological plasticity the evolution law for the plastic distortion $\boldsymbol{\beta}_{\mathrm{p}}$ is non-unique since its skew-symmetric part $\boldsymbol{\beta}^{\mathrm{skw}}$ is not defined. This situation is archetypical for phenomenological plasticity and is without consequence there, since the stresses compute only from the symmetric part of the elastic distortion. It is illuminating to note that the above evolution law for the (symmetric part of the) plastic distortion is consistent with the subdifferential $\boldsymbol{\varsigma}_{\mathrm{p}}^{\text {dev }} \in \mathfrak{d}_{\dot{\beta}_{\mathrm{p}}} D$ of the plastic potential $D=\tau_{0}\left|\dot{\boldsymbol{\beta}}_{\mathrm{p}}^{\text {sym }}\right|$ that is obtained as the Legendre transformation of the dual dissipation potential $D^{*}$.

The above generic modelling framework for geometrically linear plasticity shall be further elucidated in more detail for the specific case of single crystal plasticity in Section 3, whereby also hardening contributions will be considered.

\subsection{Incorporation of Dislocation Density}

The non-integrability of the plastic distortion $\boldsymbol{\beta}_{\mathrm{p}}$ (see Fig. 1, left), at maintained equilibrium of $\boldsymbol{\sigma}$, represents the dislocation density $\boldsymbol{\alpha}_{\mathrm{p}}$, i.e. the Nye-Kröner tensor

$$
\boldsymbol{\alpha}_{\mathrm{p}}:=\operatorname{curl} \boldsymbol{\beta}_{\mathrm{p}} \neq \mathbf{0} \quad \text { while } \quad \operatorname{div} \boldsymbol{\sigma}=\mathbf{0} .
$$

For an integrable plastic distortion $\boldsymbol{\beta}_{\mathrm{p}}$, however, e.g. for the special case that $\boldsymbol{\beta}_{\mathrm{p}}$ is spatially constant, the dislocation density $\boldsymbol{\alpha}_{\mathrm{p}}$ vanishes identically. The Nye-Kröner tensor $\boldsymbol{\alpha}_{\mathrm{p}}$ is oftentimes denoted as the geometrically necessary dislocation density, for various accounts on $\boldsymbol{\alpha}_{\mathrm{p}}$ see e.g. Kröner [37, 35], Steinmann [54], Arsenlis and Parks [3], Cermelli and Gurtin [9]. Recall that in a Cartesian coordinate system with base vectors $\boldsymbol{e}_{i}$ the curl of $\boldsymbol{\beta}_{\mathrm{p}}=\beta_{\mathrm{p} i j} \boldsymbol{e}_{i} \otimes \boldsymbol{e}_{j}$ is expressed as curl $\boldsymbol{\beta}_{\mathrm{p}}=-\beta_{\mathrm{p} i j, k} e_{j k l} \boldsymbol{e}_{i} \otimes \boldsymbol{e}_{l}$. Diagonal entries in the coefficient matrix of $\boldsymbol{\alpha}_{\mathrm{p}}$ denote the density of screw dislocations whereas the off-diagonal entries denote 
the density of edge dislocations.

Spatially non-homogeneous distributions of the plastic distortion necessitate (trapped) dislocations that have not yet left the local neighbourhood of a material point $\boldsymbol{x}$. These are oftentimes denoted as geometrically necessary dislocations and, e.g. for the case of edge dislocations, are characterized by crystal lattice planes ending in the dislocation core. In general a single dislocation is characterized by its dislocation line with tangent $t$ and the Burgers vector denoting the closure failure of a Burgers circuit $\oint_{\mathcal{C}} \boldsymbol{\beta}_{\mathrm{p}} \cdot \mathrm{d} \boldsymbol{x}$ enclosing the dislocation line.

The dislocation density $\boldsymbol{\alpha}_{\mathrm{p}}$ (containing gradients of the plastic distortion, aka 'strain gradients') contributes to the energy storage function $W$ (and eventually leads to kinematic-type hardening), whereby the corresponding variational derivative $\delta(\bullet)^{2}$ renders the 'driving force' $\varsigma_{\mathrm{p}}$ (now incorporating zeroth and second derivatives of the plastic distortion) for the evolution of the plastic distortion

$$
W=W\left(\boldsymbol{\beta}^{\mathrm{sym}}, \boldsymbol{\beta}_{\mathrm{p}}^{\mathrm{sym}}, \boldsymbol{\alpha}_{\mathrm{p}}\right) \quad \text { with } \quad \boldsymbol{\varsigma}_{\mathrm{p}}:=-\delta_{\beta_{\mathrm{p}}} W=-\partial_{\beta_{\mathrm{p}}} W-\operatorname{curl} \partial_{\alpha_{\mathrm{p}}} W .
$$

The above motivation for the direct incorporation of 'strain gradients' in the format of the dislocation density into the constitutive modelling is classical, see e.g. Steinmann [54], Menzel and Steinmann [48].

Example: A typical constitutive choice for extending the energy storage function $W$ by contributions from the dislocation density consists of a local and a gradient contribution (eventually resulting in dislocation density induced kinematic hardening)

\footnotetext{
${ }^{2}$ Recall the operational definition of the variational derivative $\delta_{\beta_{\mathrm{p}}} W^{\mathrm{grd}}$ of a function $W^{\text {grd }}=W^{\text {grd }}\left(\boldsymbol{\alpha}_{\mathrm{p}}\right)$ with $\boldsymbol{\alpha}_{\mathrm{p}}=\operatorname{curl} \boldsymbol{\beta}_{\mathrm{p}}$ via

$$
\int_{\mathcal{V}} \delta W^{\text {grd }} \mathrm{d} v=\int_{\mathcal{V}} \partial_{\alpha_{\mathrm{p}}} W^{\text {grd }}: \operatorname{curl} \delta \boldsymbol{\beta}_{\mathrm{p}} \mathrm{d} v=: \int_{\mathcal{V}} \delta_{\beta_{\mathrm{p}}} W^{\text {grd }}: \delta \boldsymbol{\beta}_{\mathrm{p}} \mathrm{d} v
$$

The variation of the functional on the left-hand-side of this definition is then reformulated via partial integration and the Gauss theorem

$$
\int_{\mathcal{V}} \partial_{\alpha_{\mathrm{p}}} W^{\text {grd }}: \operatorname{curl} \delta \boldsymbol{\beta}_{\mathrm{p}} \mathrm{d} v=\int_{\mathcal{V}} \operatorname{curl} \partial_{\alpha_{\mathrm{p}}} W^{\mathrm{grd}}: \delta \boldsymbol{\beta}_{\mathrm{p}} \mathrm{d} v+\int_{\partial \mathcal{V}}\left[\delta_{\beta_{\mathrm{p}}} W^{\mathrm{grd}} \cdot \widehat{\boldsymbol{n}}\right]: \delta \boldsymbol{\beta}_{\mathrm{p}} \mathrm{d} a
$$

whereby $\widehat{\boldsymbol{n}}$ is the skew-symmetric spin tensor corresponding to the outwards pointing normal, i.e. its axial vector $\boldsymbol{n}$ (thus for any vector $\boldsymbol{v}$ it holds isomorphically that $\widehat{\boldsymbol{n}} \cdot \boldsymbol{v}=\boldsymbol{n} \times \boldsymbol{v}$ ). Then for admissible variations of $\boldsymbol{\beta}_{\mathrm{p}}$ satisfying $\delta \boldsymbol{\beta}_{\mathrm{p}}=\mathbf{0}$ on $\partial V$ the variational derivative of $W^{\text {grd }}\left(\boldsymbol{\alpha}_{\mathrm{p}}\right)$ is eventually identified as

$$
\delta_{\beta_{\mathrm{p}}} W^{\mathrm{grd}}:=\operatorname{curl} \partial_{\alpha_{\mathrm{p}}} W^{\text {grd }} .
$$

Observe the positive sign of the curl on the right-hand-side, which is in contrast to the variational derivative of a function that is expressed in terms of the gradient of an independent variable, i.e. for $W^{\text {loc }}=W^{\text {loc }}(\boldsymbol{\beta})$ with $\boldsymbol{\beta}=\operatorname{grad} \boldsymbol{u}$ it holds that $\delta_{u} W^{\text {loc }}=-\operatorname{div} \partial_{\beta} W^{\text {loc }}$.
} 


$$
W=W\left(\boldsymbol{\beta}^{\mathrm{sym}}, \boldsymbol{\beta}_{\mathrm{p}}^{\mathrm{sym}}, \boldsymbol{\alpha}_{\mathrm{p}}\right)=W^{\mathrm{loc}}\left(\left[\boldsymbol{\beta}-\boldsymbol{\beta}_{\mathrm{p}}\right]^{\text {sym }}\right)+W^{\mathrm{grd}}\left(\boldsymbol{\alpha}_{\mathrm{p}}\right) .
$$

A popular example for the local contribution $W^{\text {loc }}$ is again the quadratic Hooke type model from Eq. 34. Moreover, with the simplest quadratic choice for the gradient contribution

$$
W^{\mathrm{grd}}=W^{\mathrm{grd}}\left(\boldsymbol{\alpha}_{\mathrm{p}}\right)=\frac{1}{2} \ell_{W}^{2} A\left|\boldsymbol{\alpha}_{\mathrm{p}}\right|^{2},
$$

the 'driving force' $\varsigma_{\mathrm{p}}$ computes eventually as

$$
\boldsymbol{\varsigma}_{\mathrm{p}}\left(\boldsymbol{\beta}, \boldsymbol{\beta}_{\mathrm{p}}, \boldsymbol{\alpha}_{\mathrm{p}}\right)=\boldsymbol{E}:\left[\boldsymbol{\beta}-\boldsymbol{\beta}_{\mathrm{p}}\right]^{\mathrm{sym}}-\ell_{W}^{2} A \operatorname{curl} \boldsymbol{\alpha}_{\mathrm{p}}
$$

The material parameter $A$ has dimension of energy per unit volume (or likewise dimension of stress) and $\ell_{W}$ is an energetic length scale.

\subsection{Incorporation of Disequilibrium Density}

Recall that the plastic stress $\sigma_{\mathrm{p}}$ (see Fig. 1, right) is a non-equilibrium stress ${ }^{3}$. Thus, the non-equilibrium source $\varrho_{\mathrm{p}}$, a novel quantity denoted the disequilibrium density, has the flavour of a body force and computes, at maintained integrability of $\boldsymbol{\beta}$, as

$$
\varrho_{\mathrm{p}}:=-\operatorname{div} \boldsymbol{\sigma}_{\mathrm{p}} \neq \mathbf{0} \text { while } \operatorname{curl} \boldsymbol{\beta}=\mathbf{0} .
$$

For a self-equilibrated plastic stress $\sigma_{\mathrm{p}}$, however, e.g. for the special case that $\boldsymbol{\sigma}_{\mathrm{p}}$ is spatially constant, the disequilibrium density $\varrho_{\mathrm{p}}$ vanishes identically. The entries in the coefficient (column) matrix of $\varrho_{\mathrm{p}}$ simply denote the disequilibrium density in the various coordinate directions.

Since we purposely avoid to introduce additional degrees of freedom, there is no thermodynamically conjugated kinematic variable to $\varrho_{\mathrm{p}}$. Thus, it is here proposed that the disequilibrium density $\varrho_{\mathrm{p}}$ (containing gradients of the plastic stress, aka 'stress gradients') parameterizes the dual dissipation potential $D^{*}$, with the rate of the (symmetric part of the) plastic distortion $\boldsymbol{\beta}_{\mathrm{p}}$ lying, as before, in the sub-differential of $D^{*}$, i.e.

$$
D^{*}=D^{*}\left(\boldsymbol{\varsigma}_{\mathrm{p}} ; \boldsymbol{\varrho}_{\mathrm{p}}\right) \quad \text { with } \quad \dot{\boldsymbol{\beta}}_{\mathrm{p}}^{\text {sym }} \in \mathfrak{d}_{\varsigma_{\mathrm{p}}} D^{*} .
$$

Recall that for rate-independent plastic solids, the convex but non-smooth dual dissipation potential $D^{*}$ is the indicator function $I$ of the admissible domain, that is now additionally parameterized in the disequilibrium density and that is expressed for phenomenological plasticity in terms of a convex yield condition

\footnotetext{
${ }^{3}$ It shall indeed be noted carefully that the plastic stress is not the residual stress! The residual stress would compute from the requirement of equilibrium after global unloading, i.e. for zero external loading data (zero body forces and boundary tractions), and would result in an integrable non-null (total) distortion $\boldsymbol{\beta} \neq \mathbf{0}$.
} 


$$
D^{*}=I\left(Y\left(\boldsymbol{\varsigma}_{\mathrm{p}} ; \boldsymbol{\varrho}_{\mathrm{p}}\right)\right) \quad \text { with } \quad Y\left(\boldsymbol{\varsigma}_{\mathrm{p}} ; \boldsymbol{\varrho}_{\mathrm{p}}\right) \leq 0 .
$$

Consequently the evolution of the (symmetric part of the) plastic distortion follows formally unchanged as in Eq. 19.

Example: For phenomenological (poly-crystalline) metal plasticity a possible yield condition in the spirit of $\mathrm{v}$. Mises extended by the influence of the disequilibrium density reads

$$
Y=Y\left(\boldsymbol{\varsigma}_{\mathrm{p}} ; \varrho_{\mathrm{p}}\right):=\left|\boldsymbol{\varsigma}_{\mathrm{p}}^{\mathrm{dev}}\right|-\tau_{0}\left[1+\ell_{D}\left|\varrho_{\mathrm{p}}\right| / \varrho_{0}\right] \leq 0 .
$$

Here $\ell_{D}$ is a dissipative length scale and the reference value $\varrho_{0}$ has dimension of stress. Similar formulations for the yield resistance, however in terms of pileup induced stress gradients have been considered by Taheri-Nassaj and Zbib [57], Lyu, Taheri-Nassay and Zbib [45], Lyu et al. [44].

\section{Application: Single Crystal Plasticity}

We will next elucidate the concepts motivated generically in the above for the specific case of geometrically linear, rate-independent single crystal plasticity. Thereby, the rate-independent case is chosen here merely for the sake of presentation, it is however remarked that accompanying issues regarding a possible indeterminacy for the active set of slip systems are entirely by-passed by resorting to the physically more appropriate rate-dependent setting (see also the computational example in Section 4).

\subsection{Local Single Crystal Plasticity}

To set the stage and to fix ideas and notation, the case of local rate-independent single crystal plasticity is first briefly reviewed. For local single crystal plasticity the plastic distortion $\boldsymbol{\beta}_{\mathrm{p}}$ results from plastic slips $\gamma_{a}$ on $a=1 \cdots n_{\text {sys }}$ slip systems (no summation convention applied here to the counter $a$ ) with slip directions $\boldsymbol{s}_{a}$ and slip plane normals $\boldsymbol{m}_{a}$ as

$$
\boldsymbol{\beta}_{\mathrm{p}}=\sum_{a} \gamma_{a} \boldsymbol{s}_{a} \otimes \boldsymbol{m}_{a}
$$

The slip direction and slip plane normal are orthonormal vectors that satisfy $\left|\boldsymbol{s}_{a}\right|=1,\left|\boldsymbol{m}_{a}\right|=1$ and $\boldsymbol{s}_{a} \cdot \boldsymbol{m}_{a}=0$. In addition the slip plane bi-normals $\boldsymbol{n}_{a}:=\boldsymbol{s}_{a} \times \boldsymbol{m}_{a}$ are introduced so that $\left\{\boldsymbol{s}_{a}, \boldsymbol{m}_{a}, \boldsymbol{n}_{a}\right\}$ form an orthonormal triad of base vectors for each slip system $a$. In the sequel the set of quantities related to all slip systems are occasionally denoted by an underline, for example the set of plastic slips is collectively denoted as

$$
\underline{\gamma}:=\left\{\gamma_{1}, \cdots, \gamma_{a}, \cdots, \gamma_{n_{\mathrm{sys}}}\right\}
$$


with the definition of the product of such sets given e.g. by $\underline{\gamma} \circ \underline{\gamma}:=\sum_{a} \gamma_{a} \gamma_{a}$.

With internal hardening variables $\eta_{a}$ for each slip system $a$ collected in the set $\underline{\eta}$, a possible quadratic energy storage function $W=W\left(\boldsymbol{\beta}, \boldsymbol{\beta}_{\mathrm{p}}, \underline{\eta}\right)$ reads as

$$
W=\frac{1}{2}[\boldsymbol{\beta}-\underbrace{\sum_{a} \gamma_{a} \boldsymbol{s}_{a} \otimes \boldsymbol{m}_{a}}_{\boldsymbol{\beta}_{\mathrm{p}}}]: \boldsymbol{E}:[\boldsymbol{\beta}-\underbrace{\sum_{b} \gamma_{b} \boldsymbol{s}_{b} \otimes \boldsymbol{m}_{b}}_{\boldsymbol{\beta}_{\mathrm{p}}}]+\frac{1}{2} \sum_{a, b} \eta_{a} H_{a b} \eta_{b} .
$$

Here, $H_{a b}=H_{b a}$ is the (symmetric) matrix of hardening moduli, allowing for self $(a=b)$ and latent $(a \neq b)$ hardening. Then, from exploiting the (isothermal) dissipation inequality

$$
\mathcal{D}:=\boldsymbol{\sigma}: \dot{\boldsymbol{\beta}}^{\mathrm{sym}}-\dot{W} \geq 0,
$$

the stress $\boldsymbol{\sigma}$ entering the equilibrium equation follows constitutively as

$$
\boldsymbol{\sigma}=\partial_{\beta} W=\boldsymbol{E}:\left[\boldsymbol{\beta}-\sum_{a} \gamma_{a} \boldsymbol{s}_{a} \otimes \boldsymbol{m}_{a}\right]^{\mathrm{sym}} .
$$

Consequently, the plastic stress $\sigma_{\mathrm{p}}$ computes upon distortion-driven unloading to $\boldsymbol{\beta}=\mathbf{0}$ as

$$
\boldsymbol{\sigma}_{\mathrm{p}}=-\boldsymbol{E}:\left[\sum_{a} \gamma_{a} \boldsymbol{s}_{a} \otimes \boldsymbol{m}_{a}\right]^{\mathrm{sym}}
$$

and results, for the sake of presentation for the - admittedly very special - case of isotropic elasticity in

$$
\boldsymbol{\sigma}_{\mathrm{p}} \stackrel{\text { isotropy }}{=}-2 G \sum_{a} \gamma_{a}\left[\boldsymbol{s}_{a} \otimes \boldsymbol{m}_{a}\right]^{\mathrm{sym}}=-G \sum_{a} \gamma_{a}\left[\boldsymbol{s}_{a} \otimes \boldsymbol{m}_{a}+\boldsymbol{m}_{a} \otimes \boldsymbol{s}_{a}\right]
$$

Next, the 'driving force' $\boldsymbol{\varsigma}_{\mathrm{p}}$ conjugate to $\boldsymbol{\beta}_{\mathrm{p}}$ computes as the negative partial derivative of the energy storage function with respect to the plastic distortion

$$
\boldsymbol{\varsigma}_{\mathrm{p}}=-\partial_{\beta_{\mathrm{p}}} W \equiv \boldsymbol{\sigma}
$$

and turns out to coincide with the stress $\sigma$ entering the equilibrium equation. Finally, the 'driving forces' $\underline{\varsigma}$ conjugate to the internal hardening variables $\underline{\eta}$ compute as

$$
\varsigma_{a}:=-\partial_{\eta_{a}} W=-\sum_{b} H_{a b} \eta_{b} .
$$

Then, with the plastic power $\boldsymbol{\varsigma}_{\mathrm{p}}: \dot{\boldsymbol{\beta}}_{\mathrm{p}}$ that expands with the definition $\tau_{a}:=$ $\boldsymbol{s}_{a} \cdot \boldsymbol{\varsigma}_{\mathrm{p}} \cdot \boldsymbol{m}_{a}$ into 


$$
\boldsymbol{\varsigma}_{\mathrm{p}}: \dot{\boldsymbol{\beta}}_{\mathrm{p}}=\sum_{a}\left[\boldsymbol{s}_{a} \cdot \boldsymbol{\varsigma}_{\mathrm{p}} \cdot \boldsymbol{m}_{a}\right] \dot{\gamma}_{a}=: \underline{\tau} \circ \underline{\dot{\gamma}},
$$

the remaining dissipation inequality reduces to a bi-linear form in terms of the 'driving forces' $\underline{\tau}$ and $\underline{\varsigma}$ and the rates of the internal variables $\underline{\gamma}$ and $\underline{\eta}$

$$
\mathcal{D}=\underline{\tau} \circ \underline{\dot{\gamma}}+\underline{\varsigma} \circ \underline{\dot{\eta}}=\sum_{a}\left[\tau_{a} \dot{\gamma}_{a}+\varsigma_{a} \dot{\eta}_{a}\right] \geq 0 .
$$

Here, $\underline{\tau}$ and $\subseteq$ denote the resolved Schmid stresses and the hardening stresses, respectively, as defined on each slip system $a$. Eventually, by comparing with the alternative representation for the remaining dissipation inequality

$$
\mathcal{D}=-\sum_{a}\left[\partial_{\gamma_{a}} W \dot{\gamma}_{a}+\partial_{\eta_{a}} W \dot{\eta}_{a}\right] \geq 0,
$$

the resolved Schmid stresses $\underline{\tau}$, i.e. the 'driving forces' conjugate to the internal variables $\underline{\gamma}$ compute as the negative partial derivatives of the energy storage function with respect to the plastic slips

$$
\tau_{a}:=-\partial_{\gamma_{a}} W \equiv \boldsymbol{s}_{a} \cdot \boldsymbol{\sigma} \cdot \boldsymbol{m}_{a} .
$$

Operationally, the resolved Schmid stress $\tau_{a}$ is thus expressed as the projection of the stress $\boldsymbol{\sigma}$ (that enters the equilibrium equation) by the Schmid tensor $\boldsymbol{s}_{a} \otimes \boldsymbol{m}_{a}$ of slip system $a$.

With the 'driving forces' in the above, for the case of rate-independent crystal plasticity (which is here chosen for the sake of simplicity), a yield condition for each slip system $a$ is formulated as

$$
Y_{a}=\left|\tau_{a}\right|-\left[\tau_{0}-\varsigma_{a}\right] \leq 0
$$

with $\tau_{0}$ the initial yield or rather slip resistance. Then, the associated evolution equations for the plastic slips $\gamma$ and the hardening variables $\eta$ follow from the constrained optimization problem $\mathcal{L}:=-\mathcal{D}+\underline{\lambda} \circ \underline{Y} \rightarrow \min _{\underline{\tau}, \underline{\subseteq}}$ as

$$
\dot{\gamma}_{a}=\lambda_{a} \partial_{\tau_{a}} Y_{a}=\lambda_{a} \frac{\tau_{a}}{\left|\tau_{a}\right|} \quad \text { and } \quad \dot{\eta}_{a}=\lambda_{a} \partial_{\varsigma_{a}} Y_{a}=\lambda_{a} .
$$

Here, $\lambda_{a} \geq 0$ are positive multipliers that satisfy, jointly with the yield conditions $Y_{a} \leq 0$, the Karush-Kuhn-Tucker optimality (or rather complementary) conditions together with the consistency conditions

$$
\lambda_{a} \geq 0, \quad Y_{a} \leq 0, \quad \lambda_{a} Y_{a}=0, \quad \text { and } \quad \lambda_{a} \dot{Y}_{a}=0 .
$$

Note that as a consequence of the above evolution equations the multipliers do here coincide with the rate of the internal hardening variables, i.e. $\lambda_{a} \equiv\left|\dot{\gamma}_{a}\right| \equiv$ $\dot{\eta}_{a}$ 
Remark: It is interesting to note that the above relations may be recast within the setting of convex analysis as outlined in the above. To this end, the dissipation potential $D=D(\underline{\dot{\gamma}}, \underline{\dot{\eta}})$ and the dual dissipation potential $D^{*}=D^{*}(\underline{\tau}, \underline{\varsigma})$ are introduced. Thereby $\bar{D}$ and $D^{*}$ are related via Legendre transformation, so that the dissipation $\mathcal{D}$ is additively composed from the dissipation and the dual dissipation potential $\mathcal{D}=D+D^{*}$. Then the evolution equations for the internal variables may alternatively be expressed in terms of sub-differentials $\mathfrak{d}(\bullet)$ (i.e. the sets of sub-derivatives) of these potentials

$$
\underline{\tau}=\mathfrak{d}_{\underline{\dot{\gamma}}} D, \quad \underline{\varsigma}=\mathfrak{d}_{\underline{\dot{\eta}}} D \quad \text { and } \quad \underline{\dot{\gamma}}=\mathfrak{d}_{\underline{\tau}} D^{*}, \quad \underline{\dot{\eta}}=\mathfrak{d}_{\underline{\varsigma}} D^{*} .
$$

Concretely, for the current setting of geometrically linear, rate-independent single crystal plasticity the dissipation potential $D=\sum_{a} D_{a}$ reads for each slip system $a$

$$
D_{a}:=\left[\tau_{0}+\sum_{b} H_{a b} \eta_{b}\right]\left|\dot{\gamma}_{a}\right|-\left[\sum_{b} H_{a b} \eta_{b}\right] \dot{\eta}_{a}
$$

with corresponding sub-differentials resulting in

$$
\tau_{a}=\left\{\begin{aligned}
+\left[\tau_{0}+\sum_{b} H_{a b} \eta_{b}\right] & & \dot{\gamma}_{a}>0 \\
\in\left[ \pm\left[\tau_{0}+\sum_{b} H_{a b} \eta_{b}\right]\right] & \text { for } & \dot{\gamma}_{a}=0 \\
-\left[\tau_{0}+\sum_{b} H_{a b} \eta_{b}\right] & & \dot{\gamma}_{a}<0
\end{aligned}\right.
$$

and

$$
\varsigma_{a}=-\sum_{b} H_{a b} \eta_{b}
$$

Observe that for $\dot{\gamma}_{a} \neq 0$ - whereby $\left|\tau_{a}\right|=\tau_{0}+\sum_{b} H_{a b} \eta_{b}$ - the above reads $\tau_{a}=\left|\tau_{a}\right| \dot{\gamma}_{a} /\left|\dot{\gamma}_{a}\right|$ and with the identification $\lambda_{a}=\left|\dot{\gamma}_{a}\right|$ corresponds thus to the evolution equations for the plastic slips as given in Eq. 46. Correspondingly, the dual dissipation potential $D^{*}=\sum_{a} D_{a}^{*}$ expands for each slip system $a$ in terms of the indicator function $I(\bullet)$ as

$$
D_{a}^{*}:=I\left(Y_{a}\right)=\left\{\begin{array}{ccc}
0 & & Y_{a} \leq 0 \\
& \text { for } & \\
\infty & & \text { else }
\end{array}\right.
$$

The evolution laws (the associated flow rules) for $\gamma_{a}$ and $\eta_{a}$ then follow as the sub-differentials

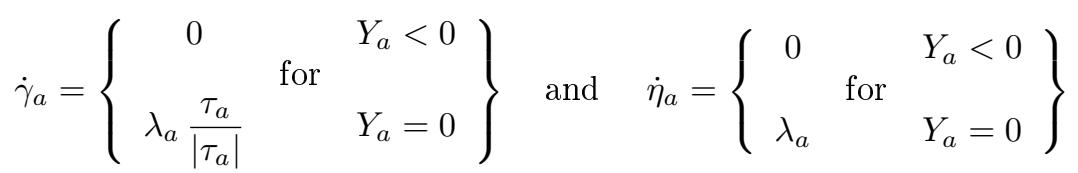

with $\lambda_{a}$ positive (plastic) multipliers. 


\subsection{Single Crystal Dislocation Density}

For the kinematics of geometrically linear single crystal plasticity the curl of the plastic distortion $\boldsymbol{\beta}_{\mathrm{p}}$ computes as

$$
\operatorname{curl} \boldsymbol{\beta}_{\mathrm{p}}=\sum_{a} \boldsymbol{s}_{a} \otimes\left[\operatorname{grad} \gamma_{a} \times \boldsymbol{m}_{a}\right] .
$$

Thus, with (scalar-valued) edge and screw dislocation densities $\alpha_{a}^{\perp}$ and $\alpha_{a}^{\|}$defined as

$$
\alpha_{a}^{\perp}:=\nabla_{s_{a}} \gamma_{a}:=\operatorname{grad} \gamma_{a} \cdot \boldsymbol{s}_{a} \quad \text { and } \quad \alpha_{a}^{\|}:=-\nabla_{n_{a}} \gamma_{a}:=-\operatorname{grad} \gamma_{a} \cdot \boldsymbol{n}_{a},
$$

the curl of the plastic distortion $\boldsymbol{\beta}_{\mathrm{p}}$ and thus the dislocation density $\boldsymbol{\alpha}_{\mathrm{p}}$ computes finally with the dislocation density tensor $\boldsymbol{\alpha}_{\mathrm{p} a}$ defined for each slip system $a$ as

$$
\operatorname{curl} \boldsymbol{\beta}_{\mathrm{p}}=: \boldsymbol{\alpha}_{\mathrm{p}}=\sum_{a} \boldsymbol{\alpha}_{\mathrm{p} a} \quad \text { with } \quad \boldsymbol{\alpha}_{\mathrm{p} a}:=\alpha_{a}^{\|} \boldsymbol{s}_{a} \otimes \boldsymbol{s}_{a}+\alpha_{a}^{\perp} \boldsymbol{s}_{a} \otimes \boldsymbol{n}_{a} .
$$

Note that in the orthonormal $\left\{\boldsymbol{s}_{a}, \boldsymbol{m}_{a}, \boldsymbol{n}_{a}\right\}$ triad of base vectors the edge and screw dislocation densities $\alpha_{a}^{\perp}$ and $\alpha_{a}^{\|}$are off-diagonal and diagonal terms, respectively, of a given slip system's contribution to $\boldsymbol{\alpha}_{\mathrm{p}}=$ curl $\boldsymbol{\beta}_{\mathrm{p}}$. Correspondingly, for edge and screw dislocations, $\boldsymbol{n}_{a}$ and $\boldsymbol{s}_{a}$, respectively, denote the tangent to the dislocation line, whereas $\alpha_{a}^{\perp} \boldsymbol{s}_{a}$ and $\alpha_{a}^{\|} \boldsymbol{s}_{a}$, respectively, denote the Burgers vector density associated with the slip system $a$. Thus a surface with normal $\boldsymbol{n}_{a}$ is penetrated by edge dislocations with Burgers vector density $\alpha_{a}^{\perp} \boldsymbol{s}_{a}$, whereas a surface with normal $\boldsymbol{s}_{a}$ is penetrated by screw dislocations with Burgers vector density $\alpha_{a}^{\|} \boldsymbol{s}_{a}$. Note, however, that in stark contrast to the aforementioned observations, for this kinematics of the plastic distortion a surface with normal $\boldsymbol{m}_{a}$ (i.e. the slip plane) is obviously not penetrated by dislocations. Finally, it shall also be noted carefully that the directional derivative of the plastic slip $\gamma_{a}$ in the direction of the slip plane normal $\boldsymbol{m}_{a}$, i.e. $\nabla_{m_{a}} \gamma_{a}$ does not contribute to the dislocation density tensor at all.

\subsection{Single Crystal Disequilibrium Density}

Assuming next for simplicity, but without loss of generality, isotropic linear elastic behaviour of the crystal lattice, the plastic stress computes explicitly as

$$
\boldsymbol{\sigma}_{\mathrm{p}}=-G \sum_{\alpha} \gamma_{a}\left[\boldsymbol{s}_{a} \otimes \boldsymbol{m}_{a}+\boldsymbol{m}_{a} \otimes \boldsymbol{s}_{a}\right] .
$$

Then the (negative) divergence of the plastic stress $\sigma_{\mathrm{p}}$ follows as

$$
-\operatorname{div} \boldsymbol{\sigma}_{\mathrm{p}}=\sum_{a}\left[G\left[\operatorname{grad} \gamma_{a} \cdot \boldsymbol{m}_{a}\right] \boldsymbol{s}_{a}+G\left[\operatorname{grad} \gamma_{a} \cdot \boldsymbol{s}_{a}\right] \boldsymbol{m}_{a}\right] .
$$


Recall next the expansion of the gradient of the plastic slip $\gamma_{a}$ in the $\left\{\boldsymbol{s}_{a}, \boldsymbol{m}_{a}, \boldsymbol{n}_{a}\right\}$ triad of base vectors and the orthonormality of $\boldsymbol{s}_{a}, \boldsymbol{m}_{a}$, and $\boldsymbol{n}_{a}$. Then (scalarvalued) disequilibrium densities $\varrho_{a}$ and $\varrho_{a}^{\uparrow}$ are defined as

$$
\varrho_{a} / G:=\nabla_{m_{a}} \gamma_{a}:=\operatorname{grad} \gamma_{a} \cdot \boldsymbol{m}_{a} \quad \text { and } \quad \varrho_{a}^{\uparrow} / G:=\nabla_{s_{a}} \gamma_{a}:=\operatorname{grad} \gamma_{a} \cdot \boldsymbol{s}_{a} \text {. }
$$

With these preliminaries at hand the (negative) divergence of the plastic stress $\sigma_{\mathrm{p}}$ and thus the disequilibrium density $\varrho_{\mathrm{p}}$ computes with the disequilibrium density vector $\varrho_{\mathrm{r} a}$ defined for each slip system $a$ as

$$
-\operatorname{div} \boldsymbol{\sigma}_{\mathrm{p}}=: \varrho_{\mathrm{p}}=\sum_{a} \varrho_{\mathrm{r} a} \quad \text { with } \quad \varrho_{\mathrm{r} a}=\varrho_{a} \boldsymbol{s}_{a}+\varrho_{a}^{\uparrow} \boldsymbol{m}_{a}
$$

Remarkably, for a given slip system $a$ the disequilibrium density consists of $\varrho_{a} \vec{a}$ in the direction of slip $s_{a}$ and of $\varrho_{a}^{\uparrow}$ in the direction of the slip plane normal $\boldsymbol{m}_{a}$. Note the identification of $\varrho_{a}^{\uparrow} \equiv G \alpha_{a}^{\perp}$ with the edge dislocation density $\alpha_{a}^{\perp}$, which is obvious in view of the subsequent example. It seems in particular that this is a force that acts in the direction of the ending lattice planes. Observe however that for this kinematics of the plastic distortion there is zero disequilibrium density in the direction $\boldsymbol{n}_{a}$ of the slip plane bi-normals. Finally, it shall also be noted carefully that the directional derivative of the plastic slip $\gamma_{a}$ in the direction of the slip plane bi-normal $\boldsymbol{n}_{a}$, i.e. $\nabla_{n_{a}} \gamma_{a}$ does not contribute to the disequilibrium density vector at all.

\subsection{Example: Edge Dislocation Induced Bending}
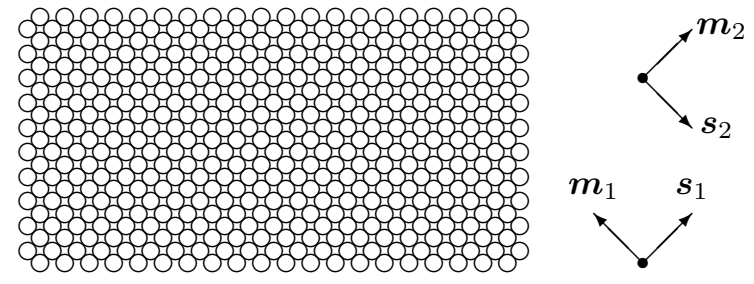

Figure 4: Idealized crystal with two slip systems that allow plastic slip along the diagonals.

As a simple two-dimensional example (bearing similarities to corresponding examples analysed e.g. by Ashby [4] and De Wit [11]) that illustrates the concepts of dislocation density and disequilibrium density, we consider the idealized crystal in Fig. 4 endowed with two slip systems characterized by the slip directions $\boldsymbol{s}_{a}$ and slip plane normals $\boldsymbol{m}_{a}$ with $a=1,2$ (and thus consequently sharing the same out-of-plane bi-normal $\boldsymbol{n}_{a}=\boldsymbol{e}_{3}$ )

$$
\left[\boldsymbol{s}_{1}\right]_{i} \propto\left[\begin{array}{c}
+1 \\
+1
\end{array}\right], \quad\left[\boldsymbol{s}_{2}\right]_{i} \propto\left[\begin{array}{c}
+1 \\
-1
\end{array}\right], \quad\left[\boldsymbol{m}_{1}\right]_{i} \propto\left[\begin{array}{c}
-1 \\
+1
\end{array}\right], \quad\left[\boldsymbol{m}_{2}\right]_{i} \propto\left[\begin{array}{c}
+1 \\
+1
\end{array}\right] .
$$

Thus, the corresponding Schmid projection tensors read 


$$
\left[\boldsymbol{s}_{1} \otimes \boldsymbol{m}_{1}\right]_{i j}=\frac{1}{2}\left[\begin{array}{cc}
-1 & +1 \\
-1 & +1
\end{array}\right] \quad \text { and } \quad\left[\boldsymbol{s}_{2} \otimes \boldsymbol{m}_{2}\right]_{i j}=\frac{1}{2}\left[\begin{array}{cc}
+1 & +1 \\
-1 & -1
\end{array}\right] .
$$

In the two-dimensional setting the only non-vanishing coefficients of the dislocation density defined by $\alpha_{\mathrm{p} i l}=-\beta_{\mathrm{p} i j, k} e_{j k l}$ are obviously

$$
\alpha_{\mathrm{p} 13}=\beta_{\mathrm{p} 12,1}-\beta_{\mathrm{p} 11,2} \quad \text { and } \quad \alpha_{\mathrm{p} 23}=\beta_{\mathrm{p} 22,1}-\beta_{\mathrm{p} 21,2} .
$$

These denote the edge dislocations with dislocation lines in $\boldsymbol{e}_{3}$-direction, i.e. penetrating the $\boldsymbol{e}_{1}-\boldsymbol{e}_{2}$ plane, and with Burgers vectors in $\boldsymbol{e}_{1}$ and $\boldsymbol{e}_{2}$ direction, respectively. The displacement field of a globally compatible bending deformation is given in terms of two parameters $c_{1}$ and $c_{2}$

$$
\boldsymbol{u}=-c_{1} x_{1} x_{2} \boldsymbol{e}_{1}+\frac{1}{2}\left[c_{1} x_{1}^{2}+c_{2} x_{2}^{2}\right] \boldsymbol{e}_{2}
$$

with the coefficients of the corresponding total distortion and corresponding dislocation density computed as

$$
[\boldsymbol{\beta}]_{i j}=\left[\begin{array}{rr}
-c_{1} x_{2} & -c_{1} x_{1} \\
c_{1} x_{1} & c_{2} x_{2}
\end{array}\right] \quad \text { with } \quad \alpha_{13}=\alpha_{23} \equiv 0 .
$$

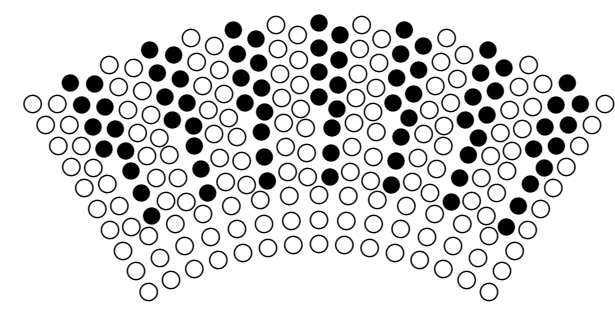

Figure 5: Macroscopically stress-free (!) distortion of an idealized crystal. The total and the plastic dislocation densities are constant. Dislocations are formed by ending lattice lines (planes) that are here marked by full circles.

For a possible macroscopically stress-free distortion (since the elastic distortion is skew symmetric) as depicted in Fig. 5 with the elastic and plastic contributions

$$
\left[\boldsymbol{\beta}_{\mathrm{e}}\right]_{i j}=\left[\begin{array}{cc}
0 & -c x_{1} \\
c x_{1} & 0
\end{array}\right] \quad \text { and } \quad\left[\boldsymbol{\beta}_{\mathrm{p}}\right]_{i j}=\left[\begin{array}{cc}
-c x_{2} & 0 \\
0 & c x_{2}
\end{array}\right],
$$

whereby an incompressible plastic distortion is obtained by setting $c_{2}=c_{1}=c$ (since then the trace of the plastic distortion vanishes), and for plastic slips $\gamma_{1}=c x_{2}$ and $\gamma_{2}=-c x_{2}$ resulting in $\alpha_{1}^{\perp}=\alpha_{2}^{\perp}=c / \sqrt{2}$ and $\alpha_{1}^{\|}=\alpha_{2}^{\|}=0$, the dislocation density takes a constant value

$$
\alpha_{\mathrm{p} 13}=c \quad \text { and } \quad \alpha_{\mathrm{p} 23}=0 .
$$

Observe that the curl of the dislocation density vanishes in this case so that the presence of the constant dislocation density is not felt as a back stress (see 
below) in the yield condition. Note furthermore that although the distortion is macroscopically stress-free, the dislocation cores at the end of the lattice lines in Fig. 5 (consisting of the five closest atoms) experience locally severe lattice distortions. Assuming next for convenience isotropic Hooke's, the plastic stress $\boldsymbol{\sigma}_{\mathrm{p}}$ and its (negative) divergence $-\operatorname{div} \boldsymbol{\sigma}_{\mathrm{p}}=: \boldsymbol{\varrho}_{\mathrm{p}}$, aka the disequilibrium density (with $\varrho_{1}^{\rightarrow}=-\varrho_{2}=\varrho_{1}^{\uparrow}=\varrho_{2}^{\uparrow}=G c / \sqrt{2}$ ) compute as

$$
\left[\boldsymbol{\sigma}_{\mathrm{r}}\right]_{i j}=-2 G\left[\begin{array}{cc}
-c x_{2} & 0 \\
0 & c x_{2}
\end{array}\right] \quad \text { and } \quad\left[\varrho_{\mathrm{r}}\right]_{i}=2 G\left[\begin{array}{l}
0 \\
c
\end{array}\right] \text {. }
$$

Thus, whereas the constant dislocation density does here not contribute to the resistance against plastic flow upon further loading, the disequilibrium density is non-zero and may thus contribute effectively to the hardening state of the material.

\subsection{Gradient Single Crystal Plasticity}

\subsubsection{Incorporation of Dislocation Density}

Recall that for single crystal plasticity the dislocation density $\boldsymbol{\alpha}_{\mathrm{p}}$ results from the plastic slip gradients grad $\gamma_{a}$ on the $a=1 \cdots n_{\text {sys }}$ slip systems as

$$
\boldsymbol{\alpha}_{\mathrm{p}}=\sum_{a} \alpha_{a}^{\|} \boldsymbol{s}_{a} \otimes \boldsymbol{s}_{a}+\alpha_{a}^{\perp} \boldsymbol{s}_{a} \otimes \boldsymbol{n}_{a} .
$$

Since the dislocation density $\boldsymbol{\alpha}_{\mathrm{p}}=\boldsymbol{\alpha}_{\mathrm{p}}\left(\underline{\alpha}^{\|}, \underline{\alpha}^{\perp} ; \underline{\boldsymbol{s}}, \underline{\boldsymbol{n}}\right)$ is a function of the screw and edge dislocation densities assembled in the sets $\underline{\alpha}^{\|}$and $\underline{\alpha}^{\perp}$, a generalized single crystal plasticity formulation is obtained by incorporating physically motivated 'strain gradients' in the format of $\underline{\alpha}^{\|}$and $\underline{\alpha}^{\perp}$ into the energy storage function $W$ that decomposes for convenience of exposition into a local and a gradient contribution

$$
W=W^{\mathrm{loc}}\left(\boldsymbol{\beta}^{\mathrm{sym}}, \underline{\gamma}, \underline{\eta} ; \underline{\boldsymbol{s}}, \underline{\boldsymbol{m}}\right)+W^{\operatorname{grd}}\left(\underline{\alpha}^{\|}, \underline{\alpha}^{\perp} ; \underline{\boldsymbol{s}}, \underline{\boldsymbol{m}}\right) .
$$

Thereby the local contribution $W^{\text {loc }}=W^{\text {loc }}\left(\boldsymbol{\beta}^{\mathrm{sym}}, \underline{\gamma}, \underline{\eta} ; \underline{\boldsymbol{s}}, \underline{\boldsymbol{m}}\right)$ to the energy storage function may expand quadratically as in Eq. $3 \overline{4}$ discussed previously for the local case. Consequently the resulting definitions of the stress $\boldsymbol{\sigma}$ entering the equilibrium equation and the plastic stress $\boldsymbol{\sigma}_{\mathrm{p}}$ are thus formally unchanged when compared to the local case.

Next, the 'driving force' $\boldsymbol{\varsigma}_{\mathrm{p}}$ conjugate to $\boldsymbol{\beta}_{\mathrm{p}}$ computes as the negative variational derivative of the energy storage function with respect to the plastic distortion

$$
\boldsymbol{\varsigma}_{\mathrm{p}}=-\delta_{\beta_{\mathrm{p}}} W \equiv \boldsymbol{\varsigma}_{\mathrm{p}}^{\mathrm{loc}}+\boldsymbol{\varsigma}_{\mathrm{p}}^{\mathrm{grd}} .
$$

Note that as a consequence of the decomposition of the energy storage function into local and gradient contributions, the 'driving force' $\varsigma_{\mathrm{p}}$ decomposes likewise into local $\varsigma_{\mathrm{p}}^{\text {loc }}$ and gradient contributions $\varsigma_{\mathrm{p}}^{\mathrm{grd}}$. Thereby, $\varsigma_{\mathrm{p}}^{\text {loc }}$ is formally unchanged when compared to the local case 


$$
\varsigma_{\mathrm{p}}^{\mathrm{loc}}:=-\partial_{\beta_{\mathrm{p}}} W^{\mathrm{loc}} \equiv \boldsymbol{\sigma},
$$

and turns out to coincide with the stress $\boldsymbol{\sigma}$ entering the equilibrium equation. Moreover, $\varsigma_{\mathrm{p}}^{\mathrm{grd}}$ expands as

$$
\varsigma_{\mathrm{p}}^{\mathrm{grd}}:=-\operatorname{curl} \partial_{\alpha_{\mathrm{p}}} W^{\mathrm{grd}} .
$$

Finally, the 'driving forces' $\underline{\varsigma}$ conjugate to the internal hardening variables $\underline{\eta}$ compute as in the local case.

Then, with the plastic power $\boldsymbol{\varsigma}_{\mathrm{p}}: \dot{\boldsymbol{\beta}}_{\mathrm{p}}$ that expands with the definition $\tau_{a}:=$ $\boldsymbol{s}_{a} \cdot \boldsymbol{s}_{\mathrm{p}} \cdot \boldsymbol{m}_{a}$ into $\underline{\tau} \circ \dot{\gamma}$, the remaining dissipation inequality reduces to a bilinear form in terms of the 'driving forces' $\underline{\tau}$ and $\varsigma$ and the rates of the internal variables $\underline{\gamma}$ and $\eta$ formally as in Eq. 42. Note again that as a consequence of the decomposition of the energy storage function into local and gradient contributions, the Schmid stresses $\underline{\tau}$ decompose likewise into local and gradient contributions

$$
\tau_{a}=\tau_{a}^{\mathrm{loc}}+\tau_{a}^{\mathrm{grd}}
$$

Eventually, by comparing with the alternative representation for the remaining dissipation inequality

$$
\mathcal{D}=-\sum_{a}\left[\delta_{\gamma_{a}} W \dot{\gamma}_{a}+\partial_{\eta_{a}} W \dot{\eta}_{a}\right] \geq 0
$$

the resolved Schmid stresses $\underline{\tau}$, i.e. the 'driving forces' conjugate to the internal variables $\gamma$ compute as the negative variational derivatives of the energy storage function with respect to the plastic slips

$$
\tau_{a}:=-\delta_{\gamma_{a}} W=\tau_{a}^{\mathrm{loc}}+\tau_{a}^{\mathrm{grd}}=\tau_{a}^{\mathrm{loc}}-\tau_{a}^{\|}-\tau_{a}^{\perp} .
$$

Here (scalar-valued) edge and screw resolved Schmid back stresses $\underline{\tau}^{\perp}$ and $\underline{\tau}^{\|}$ resulting in $\underline{\tau}^{\text {grd }}:=-\underline{\tau}^{\|}-\underline{\tau}^{\perp}$ are defined as

$$
\tau_{a}^{\perp}:=\nabla_{s_{a}} \mu_{a}^{\perp}:=\operatorname{grad} \mu_{a}^{\perp} \cdot \boldsymbol{s}_{a} \quad \text { and } \quad \tau_{a}^{\|}:=-\nabla_{n_{a}} \mu_{a}^{\|}:=-\operatorname{grad} \mu_{a}^{\|} \cdot \boldsymbol{n}_{a}
$$

whereas the (local and gradient) 'driving forces' $\underline{\tau}^{\text {loc }}, \underline{\mu}^{\|}$and $\underline{\mu}^{\perp}$ conjugate to the internal variables $\underline{\gamma}, \underline{\alpha}^{\|}$and $\underline{\alpha}^{\perp}$, respectively, are introduced as

$$
\tau_{a}^{\mathrm{loc}}:=-\partial_{\gamma_{a}} W^{\mathrm{loc}} \quad \text { and } \quad \mu_{a}^{\|}:=-\partial_{\alpha_{a}^{\|}} W^{\text {grd }} \quad \text { and } \quad \mu_{a}^{\perp}:=-\partial_{\alpha_{a}^{\perp}} W^{\text {grd }} .
$$

Note the similar definition of the edge and screw dislocation densities and the edge and screw resolved Schmid backstresses. As net result, directional derivatives of the plastic slips in the directions $\boldsymbol{s}_{a}$ and $\boldsymbol{n}_{a}$ contribute evenly to the resolved Schmid backstresses. Operationally, the resolved Schmid stress $\tau_{a}$ is 
thus expressed as the projection of the stress $\sigma$ (that enters the equilibrium equation) by the Schmid tensor $\boldsymbol{s}_{a} \otimes \boldsymbol{m}_{a}$ of slip system $a$ and the (gradients of) derivatives of the energy storage function with respect to the (scalar-valued) screw and edge dislocation densities

$$
\tau_{a}=-\delta_{\gamma_{a}} W \equiv \boldsymbol{s}_{a} \cdot \boldsymbol{\sigma} \cdot \boldsymbol{m}_{a}-\operatorname{grad} \partial_{\alpha_{a}^{\|}} W \cdot \boldsymbol{n}_{a}+\operatorname{grad} \partial_{\alpha_{a}^{\perp}} W \cdot \boldsymbol{s}_{a} .
$$

\subsubsection{Incorporation of Disequilibrium Density}

Recall next that for single crystal plasticity (in combination with isotropic linear elasticity) the disequilibrium density $\varrho_{\mathrm{p}}$ results from the shear modulus weighted plastic slip gradients grad $\gamma_{a}$ on the $a=1 \cdots n_{\text {sys }}$ slip systems as

$$
\varrho_{\mathrm{p}}=\sum_{a} \varrho_{a}^{\vec{s}} \boldsymbol{s}_{a}+\varrho_{a}^{\uparrow} \boldsymbol{m}_{a}
$$

Since the (vector-valued) disequilibrium density $\varrho_{\mathrm{r}}=\varrho_{\mathrm{r}}\left(\varrho^{\rightarrow}, \varrho^{\uparrow} ; \underline{\boldsymbol{s}}, \underline{\boldsymbol{m}}\right)$ is a function of the (scalar-valued) disequilibrium densities assembled in the sets $\underline{\varrho} \rightarrow$ and $\underline{\varrho}^{\uparrow}$, a generalized single crystal plasticity formulation is obtained by incorporating continuum mechanics-motivated 'stress gradients' in the format of $\underline{\varrho} \rightarrow$ and $\underline{\varrho}^{\uparrow}$ (depending for isotropic linear elasticity on the shear modulus weighted plastic slip gradients $G \nabla_{m_{a}} \gamma_{a}=: \varrho_{a}^{\rightarrow}$ and $G \nabla_{s_{a}} \gamma_{a}=: \varrho_{a}^{\uparrow}$ ) as parameters in the yield conditions $\underline{Y}$ that are otherwise functions of the 'driving forces' $\underline{\tau}$ and $\underline{\varsigma}$

$$
Y_{a}=Y_{a}\left(\tau_{a}, \varsigma_{a} ; \underline{\varrho}^{\rightarrow}, \underline{\varrho}^{\uparrow}\right) .
$$

A particular obvious choice for the explicit parametrization of the yield conditions $\underline{Y}$ in the disequilibrium density $\varrho_{\mathrm{r}}$ is given by the projection

$$
\varrho_{a}:=\varrho_{\mathrm{p}} \cdot \boldsymbol{s}_{a}=\sum_{b} \varrho_{\mathrm{p} b} \cdot \boldsymbol{s}_{a}
$$

of $\varrho_{\mathrm{p}}$ into the slip directions $\boldsymbol{s}_{a}$ to render

$$
Y_{a}=\left|\tau_{a}\right|-\left[\tau_{0}\left[1+\ell_{D}\left|\varrho_{a}\right| / \varrho_{0}\right]-\varsigma_{a}\right] \leq 0
$$

with $\tau_{0}$ the initial slip resistance and $\varrho_{0}=G$ or $\varrho_{0}=\tau_{0}$ a possible reference value of dimension stress. A furthermore reduced alternative model is obtained by only using the projection

$$
\varrho_{a}:=\varrho_{\mathrm{p} a} \cdot \boldsymbol{s}_{a}
$$

of the slip-system-wise disequilibrium density $\varrho_{\mathrm{p} a}$ into the slip directions $\boldsymbol{s}_{a}$ to render

$$
Y_{a}=\left|\tau_{a}\right|-\left[\tau_{0}\left[1+\ell_{D}\left|\varrho_{a}\right| / \varrho_{0}\right]-\varsigma_{a}\right] \leq 0 .
$$

Note that $\varrho_{a}$ contributes the directional slip gradient $\nabla_{m_{a}} \gamma_{a}$ into the yield resistance, whereas $\tau_{a}$ contributes the directional slip gradients $\nabla_{s_{a}} \gamma_{a}$ and $\nabla_{n_{a}} \gamma_{a}$ 
into the equivalent stress, thus all directional slip gradients are present in the definition of the yield condition.

Regardless of the concrete modelling option, the associated evolution equations for $\gamma$ and $\eta$ follow formally as unchanged compared to Eq. 46 from the constrained optimization problem $\mathcal{L}:=-\mathcal{D}+\underline{\lambda} \circ \underline{Y} \rightarrow \min _{\underline{\tau}, \varsigma}$ whereby, again, positive multipliers $\lambda_{a} \geq 0$ satisfy, jointly with the yield conditions $Y_{a} \leq 0$, the Karush-Kuhn-Tucker optimality conditions as in Eq. 47. Note again that as a consequence of the above evolution equations the multipliers do here coincide with the rate of the hardening variables $\lambda_{a} \equiv\left|\dot{\gamma}_{a}\right| \equiv \dot{\eta}_{a}$.

Remark: In alignment with the early proposal in [54, 48] it can be considered a benefit of this particular formulation that it does not introduce additional degrees of freedom in the continuum setting that need to be solved from additional balance equations as, e.g., micro force balances à la Gurtin [21]. Nevertheless, since the gradients of the plastic slips enter the yield condition, in a computational setting such as the finite element method these need to be determined numerically in one way or another. One possibility consists in numerically solving the evolution equations in weak form, which entails discretising the plastic slips and requires the determination of the active plastic domain in an iterative fashion. Liebe et al. [38, 39], for example, have devised such algorithms earlier. An alternative is to use a sequence of $\mathrm{L}_{2}$ smoothing steps, see Menzel et al. [47].

\section{Computational Example: Micro Wire Torsion}

The effects of considering the dislocation density and the disequilibrium density within the yield condition shall be investigated by a simple computational example. To this end, we consider torsion of a (three-dimensional) single crystalline micro wire with fcc crystal structure. The finite element solution for the equilibrium boundary value problem is obtained, based on a discretisation via tri-linear Ansatz functions, by an implementation into the open source finite element library deal.ii [2]. The rate-independent model proposed in Section 3.5 is here considered in a regularized fashion, thus resulting in the rate-dependent approximation to Eq. 85 (including the gradient extended Schmid stress in Eq. 76 with $\tau_{a}^{\|}=-\ell_{W}^{2} G \nabla^{2} \gamma_{a}:\left[\boldsymbol{n}_{a} \otimes \boldsymbol{n}_{a}\right]$ and $\tau_{a}^{\perp}=-\ell_{W}^{2} G \nabla^{2} \gamma_{a}:\left[\boldsymbol{s}_{a} \otimes \boldsymbol{s}_{a}\right]$ but neglecting the contribution of the internal variables to the hardening in order to exclusively focus on the gradient effects)

$$
\dot{\gamma}_{a}=\dot{\gamma}_{0}\left[\frac{\left|\tau_{a}^{\mathrm{loc}}-\tau_{a}^{\|}-\tau_{a}^{\perp}\right|}{\tau_{0}\left[1+\ell_{D}\left|\rho_{a}\right| / \varrho_{0}\right]}\right]^{p} \operatorname{sign}\left(\tau_{a}^{\mathrm{loc}}-\tau_{a}^{\|}-\tau_{a}^{\perp}\right) .
$$

The corresponding material parameters used in our computations are assembled in Tab. 1, whereby the influence of the energetic and the dissipative length scale 
parameters $\ell_{W}$ and $\ell_{D}$ shall be analysed. The reference slip rate $\dot{\gamma}_{0}$ and the exponent $p$ are chosen so as to adjust the formulation closely to the rate-independent limit (to verify this assertion we checked that doubling the displacement-driven load rate increases the resultant torque of the following example by only $3.5 \%$ ). The crystallographic slips are evaluated at the quadrature points and subsequently smoothed using a $\mathrm{L}_{2}$ projection with linear Ansatz functions and the same mesh as for the displacement field. To obtain the necessary second gradients of the slips, the projected gradients of the slip fields are smoothed again using the same Ansatz. The gradient contributions are integrated explicitly along the load history, whereas the local crystal plasticity contributions are integrated implicitly.

\begin{tabular}{|ll|ll|ll|}
\hline $\mathrm{K}$ & $217000 \mathrm{MPa}$ & $\tau_{0}$ & $200 \mathrm{MPa}$ & $p$ & 20 \\
$\mathrm{G}$ & $100000 \mathrm{MPa}$ & $\varrho_{0}$ & $200 \mathrm{MPa}$ & $\dot{\gamma}_{0}$ & $100000 \mathrm{~s}^{-1}$ \\
\hline
\end{tabular}

Table 1: Material parameters for the micro wire torsion problem

The micro wire is loaded in torsion by rotation control, whereby inextensibility is enforced in axial direction. The displacement of the rotational axis is completely fixed to prevent rigid body motions. Moreover, the rotation of the bottom cross section is fully restricted, however radial displacements are free due to appropriately constraining the Cartesian in-plane displacements. The top cross section is rotated by a twist angle of in total $5 \mathrm{~mm}^{-1}$ (per axial length of the micro wire) with a rate of $40 \mathrm{~mm}^{-1} \mathrm{~s}^{-1}$ prescribed in 30 equidistant load/time steps for all nodes whereby the radial displacements are again free. The micro wire has a diameter of $10 \mu \mathrm{m}$ and (due to the repeatability of the solution along the axial direction) only a slice of length $0.368 \mu \mathrm{m}$ is discretised, resulting in a mesh with nearly cube-shaped elements see Fig. 6 . Due to the axial inextensibility condition one element in axial direction is sufficient, thus resulting in a total number of 320 elements. The orientation of the crystallographic unit cells in relation to the micro wire is depicted in Fig. 7 with the micro wire cross section shown in yellow and the four slip planes in green, each with its three slip systems in red.
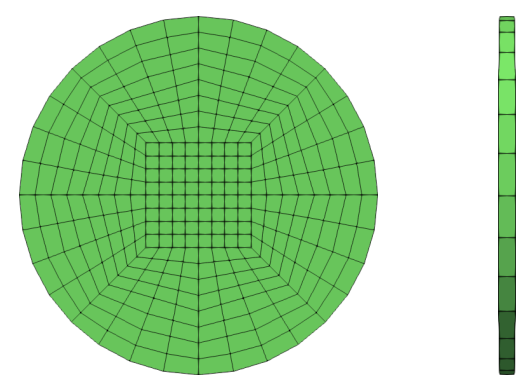

Figure 6: Mesh of a slice of the micro wire as loaded in torsion. 

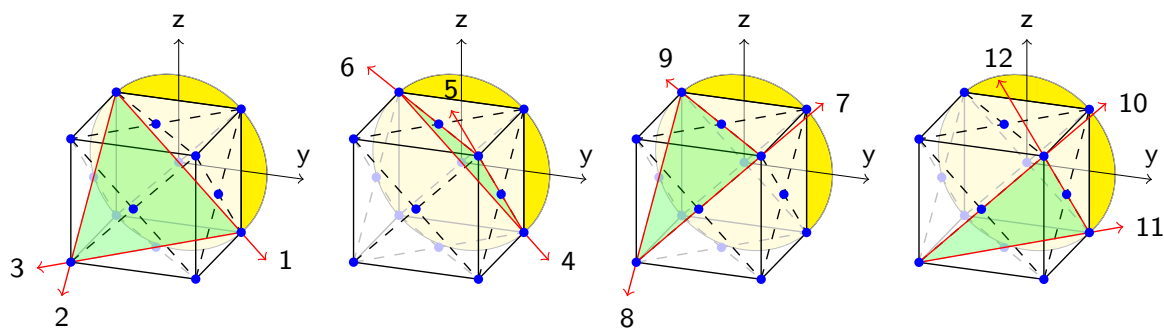

Figure 7: Four slip planes of a fcc unit cell marked in green with the corresponding three slip systems indicated as arrows. The micro wire cross section is indicated in yellow.

The micro wire torsion problem is then evaluated for different values of the energetic and dissipative length scale parameters $\ell_{W}$ and $\ell_{D}$. The resulting size-dependent hardening is highlighted in the torque - twisting diagram in Fig. 8. Thereby, the additional hardening contribution due to the disequilibrium density is activated by setting $\ell_{D} \neq 0$ and is clearly visible in the dashed lines of the zoom-out in Fig. 9 (the results obtained by only activating the hardening due to the dislocation density, i.e. for $\ell_{W} \neq 0$ and $\ell_{D}=0$ are highlighted by the full lines.

Observe that both, the inclusion of the dislocation density as well as of the disequilibrium density merely influences the hardening behaviour while leaving the initial yield limit unaffected. This does, however, not come as a surprise, since any gradient plasticity formulation that rests on some sort of gradient of the plastic distortion cannot result in an increase of the initial yield limit, but can only contribute to the hardening once plastic distortion starts evolving. It is precisely the contribution to hardening due to the novel disequilibrium density that is the main focus of this manuscript. The initial yield limit can only be influenced by some sort of gradient of the equilibrium stress (or alternatively the total distortion). This issues will be discussed in much detail shortly in a separate contribution.

The spatial distributions of the norm of the dislocation and disequilibrium densities defined as $\sqrt{\underline{\alpha}^{\perp} \circ \underline{\alpha}^{\perp}+\underline{\alpha}^{\|} \circ \underline{\alpha}^{\|}}$and $\sqrt{\underline{\varrho} \rightarrow \circ \underline{\varrho} \rightarrow+\underline{\varrho}^{\uparrow} \circ \underline{\varrho} \underline{\varrho}^{\uparrow}}$ as well as the von Mises stress for various combinations of the energetic and dissipative length scale parameters $\ell_{W}$ and $\ell_{D}$ are showcased for the sake of completeness in the Appendix in Figs. 10, 11 and 12. Likewise, the projections of the slip gradients in the directions $\boldsymbol{s}_{a}, \boldsymbol{n}_{a}$ and $\boldsymbol{m}_{a}$ at maximum twisting are depicted for the energetic and dissipative length scale parameters $\ell_{W}=0.14$ and $\ell_{D}=0.1$ in Figs. 13,14 , and 15 , respectively. 


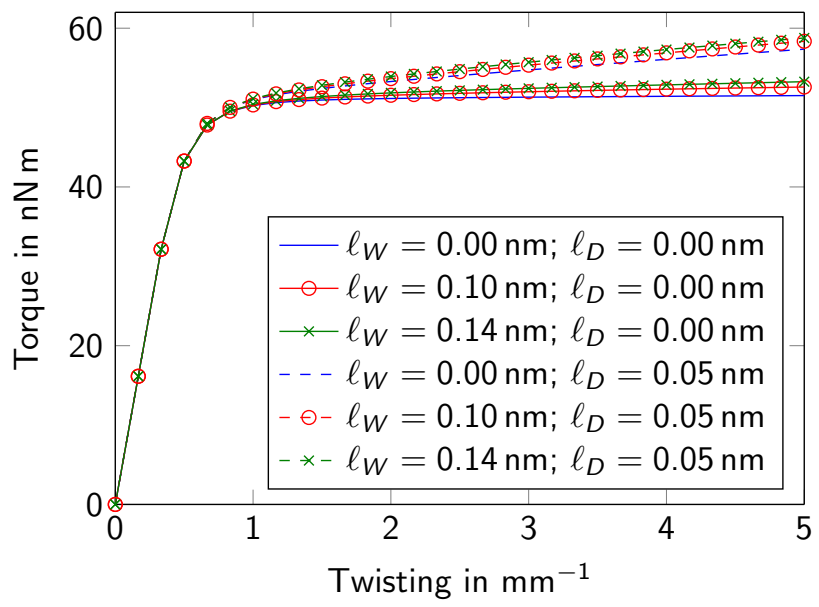

Figure 8: Torque over twisting for different values of the length scale parameters $\ell_{W}$ and $\ell_{D}$.

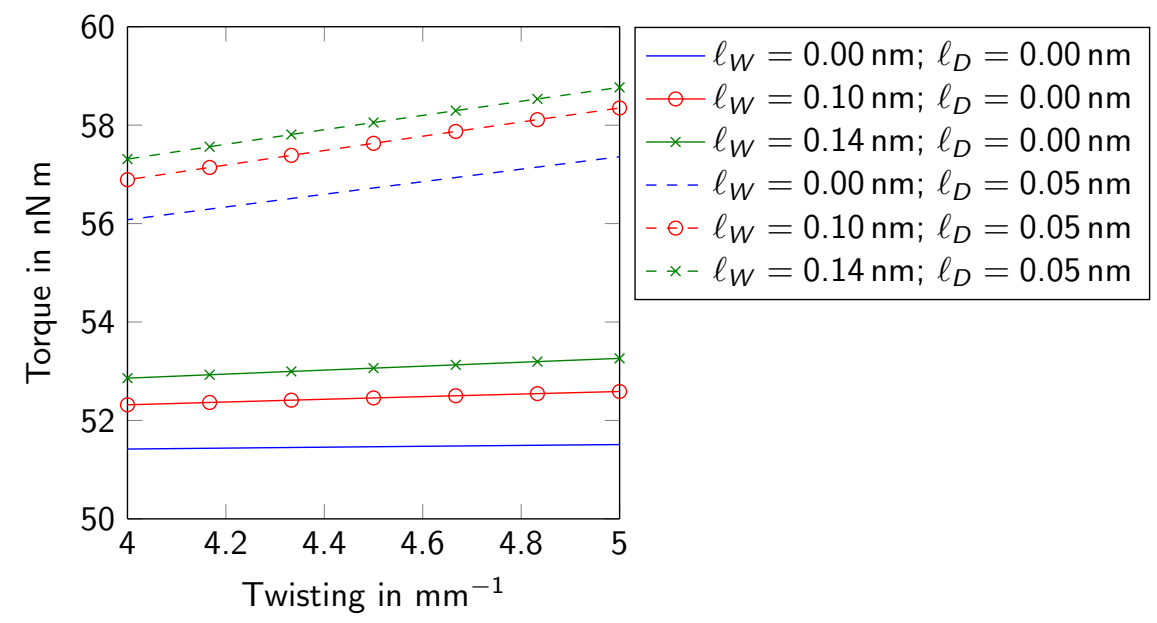

Figure 9: Zoom: Torque over twisting for different values of the length scales parameters $\ell_{W}$ and $\ell_{D}$.

\section{Conclusions}

We here propose an alternative continuum mechanics-inspired approach to 'stress gradient' plasticity that rests on the introduction of the plastic stress and its non-equilibrium that is expressed in terms of the disequilibrium density, a novel concept. In this regard our new approach is entirely different from the established format of 'stress gradient' plasticity that is rather motivated from the consideration of dislocation pileup problems. 
The here proposed novel kinetic quantities for 'stress gradient' plasticity are the counterparts to the well-established kinematic quantities in 'strain gradient' plasticity, i.e. the plastic distortion and its non-integrability expressed in terms of the dislocation density (or the plastic strain and its incompatibility expressed in terms of the incompatibility density). Indeed, we conceptually introduce the plastic stress by distortion-driven unloading from a loaded state in the elastoplastic regime in an entirely dual fashion to the accepted introduction of the (symmetric part of the) plastic distortion that commonly follows from a stressdriven unloading from the same state, see Fig. 1. Equilibrium of stress and integrability of distortion as expressed by the differential operators div and curl are complementing requirements in continuum mechanics. Thus the absence of equilibrium, i.e. the non-equilibrium of the plastic stress, as well as the absence of integrability, i.e. the non-integrability of the plastic distortion motivate us to consider these quantities in a formulation of gradient plasticity.

Thereby, the formulation of a gradient plasticity formulation is most elegantly effected within the abstract setting of convex analysis that, for the case of plasticity, hinges on a (poly-)convex energy storage function and a convex dissipation potential as well as its dual.

In our setting, the dislocation and disequilibrium densities contribute to the formulation of gradient plasticity combining 'strain' and 'stress gradients' via their introduction into the energy storage function and the dual dissipation potential. When translating the convex analysis approach to the more classical concept of a yield condition, the former results in a size-dependent back-stress like contribution to the equivalent stress, whereas the latter results in a size-dependent contribution to the yield resistance.

Noteworthy, when specified to the case of gradient single crystal plasticity combining 'strain' and 'stress gradients', all of the three independent directional derivatives of the plastic slip, i.e. in the slip direction (1) and in the directions of the slip plane bi-normal (2) and slip plane normal (3), occur. The first two directional gradients of the plastic slip typically contribute to the dislocation density in the form of (scalar-valued) edge and screw dislocation densities. The third directional gradient is usually not present in common 'strain gradient' single crystal plasticity. An exception is the contribution by Bardella and Giacomini [5] that phenomenologically introduces a dissipative higher-order stress conjugate to this uncommon directional derivative of the plastic slip within a micro-force balance setting (for an analysis of this type of gradient plasticity models see Mc Bride et al. [46]). It appears however that only the here advocated novel 'stress gradient' contribution in the form of a (scalar-valued) disequilibrium density rationalises this approach for a setting not involving an additional micro-force balance and the accompanying additional degrees of freedom. 
As a conclusion, the novel continuum approach to gradient single crystal plasticity combining 'strain' and 'stress gradients' involves the entire set of plastic slip gradients and thus allows to phenomenologically (i.e. in a continuum mechanics setting) model the size-dependent hardening state of a single crystalline material in a more complete fashion.

Detailed implications for the algorithmic setting of gradient single crystal plasticity combining 'strain' and 'stress gradients' as well as the extension to the geometrically non-linear case will be pursued in our forthcoming contributions.

\section{Acknowledgments}

PS gratefully acknowledges support by the German Science Foundation through the project P10 of the Priority Programme 2013 on 'Residual Stresses' and the project C5 of the Collaborative Research Centre 814 on 'Additive Manufacturing' as well as by the Royal Society, UK, through a Wolfson Research Merit Award.

\section{References}

[1] S. Akarapu and J.P. Hirth. Dislocation pile-ups in stress gradients revisited. Acta Materialia, 61(10):3621-3629, 2013.

[2] D. Arndt, W. Bangerth, T. C. Clevenger, D. Davydov, M. Fehling, D. Garcia-Sanchez, G. Harper, T. Heister, L. Heltai, M. Kronbichler, R. M. Kynch, M. Maier, J.-P. Pelteret, B. Turcksin, and D. Wells. The deal.II library, version 9.1. Journal of Numerical Mathematics, 2019. accepted.

[3] A. Arsenlis and D.M. Parks. Crystallographic aspects of geometricallynecessary and statistically-stored dislocation density. Acta materialia, 47(5):1597-1611, 1999.

[4] M.F. Ashby. The deformation of plastically non-homogeneous materials. The Philosophical Magazine: A Journal of Theoretical Experimental and Applied Physics, 21(170):399-424, 1970.

[5] L. Bardella and A. Giacomini. Influence of material parameters and crystallography on the size effects describable by means of strain gradient plasticity. Journal of the Mechanics and Physics of Solids, 56(9):2906-2934, 2008.

[6] B.A. Bilby, R. Bullough, and E. Smith. Continuous distributions of dislocations: A new application of the methods of non-Riemannian geometry. Proceedings of the Royal Society of London. Series A. Mathematical and Physical Sciences, 231(1185):263-273, 1955. 
[7] E. Bitzek and P. Gumbsch. Atomistic study of drag, surface and inertial effects on edge dislocations in face-centered cubic metals. Materials Science and Engineering A, 387-389:11-15, 2004.

[8] E. Bitzek and P. Gumbsch. Dynamic aspects of dislocation motion: Atomistic simulations. Materials Science and Engineering A, 400-401:40-44, 2005.

[9] P. Cermelli and M.E. Gurtin. On the characterization of geometrically necessary dislocations in finite plasticity. Journal of the Mechanics and Physics of Solids, 49(7):1539-1568, 2001.

[10] S.S. Chakravarthy and W.A. Curtin. Stress-gradient plasticity. Proceedings of the National Academy of Sciences of the United States of America, 108(38):15716-15720, 2011.

[11] R. De Wit. A view of the relation between the continuum theory of lattice defects and non-euclidean geometry in the linear approximation. International Journal of Engineering Science, 19(12):1475-1506, 1981.

[12] D.J. Dunstan, B. Ehrler, R. Bossis, S. Joly, K.M.Y. P'Ng, and A.J. Bushby. Elastic limit and strain hardening of thin wires in torsion. Physical Review Letters, 103(15), 2009.

[13] B. Ehrler, X.D. Hou, T.T. Zhu, K.M.Y. P'Ng, C.J. Walker, A.J. Bushby, and D.J. Dunstan. Grain size and sample size interact to determine strength in a soft metal. Philosophical Magazine, 88(25):3043-3050, 2008.

[14] A.G. Evans and J.W. Hutchinson. A critical assessment of theories of strain gradient plasticity. Acta Materialia, 57(5):1675-1688, 2009.

[15] N.A. Fleck and J.W. Hutchinson. Strain gradient plasticity. Advances in Applied Mechanics, 33(C):295-361, 1997.

[16] N.A. Fleck, G.M. Muller, M.F. Ashby, and J.W. Hutchinson. Strain gradient plasticity: Theory and experiment. Acta Metallurgica Et Materialia, 42(2):475-487, 1994.

[17] S. Forest. Micromorphic approach for gradient elasticity, viscoplasticity, and damage. Journal of Engineering Mechanics, 135(3):117-131, 2009.

[18] S. Forest and E.C. Aifantis. Some links between recent gradient thermoelasto-plasticity theories and the thermomechanics of generalized continua. International Journal of Solids and Structures, 47(25-26):3367-3376, 2010.

[19] S. Forest and K. Sab. Stress gradient continuum theory. Mechanics Research Communications, 40:16-25, 2012.

[20] P. Gudmundson. A unified treatment of strain gradient plasticity. Journal of the Mechanics and Physics of Solids, 52(6):1379-1406, 2004. 
[21] M.E. Gurtin. On the plasticity of single crystals: Free energy, microforces, plastic-strain gradients. Journal of the Mechanics and Physics of Solids, 48(5):989-1036, 2000.

[22] M.E. Gurtin. A gradient theory of single-crystal viscoplasticity that accounts for geometrically necessary dislocations. Journal of the Mechanics and Physics of Solids, 50(1):5-32, 2002.

[23] M.E. Gurtin. A gradient theory of small-deformation isotropic plasticity that accounts for the burgers vector and for dissipation due to plastic spin. Journal of the Mechanics and Physics of Solids, 52(11):2545-2568, 2004.

[24] M.E. Gurtin. A finite-deformation, gradient theory of single-crystal plasticity with free energy dependent on densities of geometrically necessary dislocations. International Journal of Plasticity, 24(4):702-725, 2008.

[25] M.E. Gurtin. A finite-deformation, gradient theory of single-crystal plasticity with free energy dependent on the accumulation of geometrically necessary dislocations. International Journal of Plasticity, 26(8):1073-1096, 2010.

[26] M.E. Gurtin and L. Anand. A theory of strain-gradient plasticity for isotropic, plastically irrotational materials. Part i: Small deformations. Journal of the Mechanics and Physics of Solids, 53(7):1624-1649, 2005.

[27] M.E. Gurtin and L. Anand. A theory of strain-gradient plasticity for isotropic, plastically irrotational materials. Part ii: Finite deformations. International Journal of Plasticity, 21(12):2297-2318, 2005.

[28] M.E. Gurtin and L. Anand. Thermodynamics applied to gradient theories involving the accumulated plastic strain: The theories of Aifantis and Fleck and Hutchinson and their generalization. Journal of the Mechanics and Physics of Solids, 57(3):405-421, 2009.

[29] M.E. Gurtin, L. Anand, and S.P. Lele. Gradient single-crystal plasticity with free energy dependent on dislocation densities. Journal of the $\mathrm{Me}$ chanics and Physics of Solids, 55(9):1853-1878, 2007.

[30] M.E. Gurtin and B.D. Reddy. Gradient single-crystal plasticity within a Mises-Hill framework based on a new formulation of self- and latenthardening. Journal of the Mechanics and Physics of Solids, 68(1):134-160, 2014.

[31] E.O. Hall. The deformation and ageing of mild steel: III discussion of results. Proceedings of the Physical Society. Section B, 64(9):747, 1951.

[32] W. Han and B.D. Reddy. Plasticity: mathematical theory and numerical analysis. Springer Science \& Business Media, 2012. 
[33] J.P. Hirth. Dislocation pileups in the presence of stress gradients. Philosophical Magazine, 86(25-26):3959-3963, 2006.

[34] J.P. Hirth, M. Rhee, and H. Zbib. Modeling of deformation by a 3d simulation of multiple, curved dislocations. Journal of Computer-Aided Materials Design, 3(1-3):164-166, 1996.

[35] E. Kröner. Allgemeine Kontinuumstheorie der Versetzungen und Eigenspannungen. Archive for Rational Mechanics and Analysis, 4(1):273, 1959.

[36] E. Kröner and A. Seeger. Nicht-lineare Elastizitätstheorie der Versetzungen und Eigenspannungen. Archive for Rational Mechanics and Analysis, 3(1):97-119, 1959.

[37] Ekkehart Kröner. Kontinuumstheorie der Versetzungen und Eigenspannungen. Springer, 1958.

[38] T. Liebe and P. Steinmann. Theory and numerics of a thermodynamically consistent framework for geometrically linear gradient plasticity. International Journal for Numerical Methods in Engineering, 51(12):1437-1467, 2001.

[39] T. Liebe, P. Steinmann, and A. Benallal. Theoretical and computational aspects of a thermodynamically consistent framework for geometrically linear gradient damage. Computer Methods in Applied Mechanics and Engineering, 190(49-50):6555-6576, 2001.

[40] D. Liu, Y. He, D.J. Dunstan, B. Zhang, Z. Gan, P. Hu, and H. Ding. Toward a further understanding of size effects in the torsion of thin metal wires: An experimental and theoretical assessment. International Journal of Plasticity, 41:30-52, 2013.

[41] D. Liu, Y. He, X. Tang, H. Ding, P. Hu, and P. Cao. Size effects in the torsion of microscale copper wires: Experiment and analysis. Scripta Materialia, 66(6):406-409, 2012.

[42] D. Liu, Y. He, and B. Zhang. Towards a further understanding of dislocation pileups in the presence of stress gradients. Philosophical Magazine, 93(18):2340-2362, 2013.

[43] D. Liu, Y. He, B. Zhang, and L. Shen. A continuum theory of stress gradient plasticity based on the dislocation pile-up model. Acta Materialia, 80:350$364,2014$.

[44] H. Lyu, M. Hamid, A. Ruimi, and H.M. Zbib. Stress/strain gradient plasticity model for size effects in heterogeneous nano-microstructures. International Journal of Plasticity, 97:46-63, 2017. 
[45] H. Lyu, N. Taheri-Nassaj, and H.M. Zbib. A multiscale gradient-dependent plasticity model for size effects. Philosophical Magazine, 96(18):1883-1908, 2016.

[46] A.T. McBride, B.D. Reddy, and P. Steinmann. Dissipation-consistent modelling and classification of extended plasticity formulations. Journal of the Mechanics and Physics of Solids, 119:118-139, 2018.

[47] A. Menzel, R. Denzer, and P. Steinmann. On the comparison of two approaches to compute material forces for inelastic materials. application to single-slip crystal-plasticity. Computer Methods in Applied Mechanics and Engineering, 193(48-51):5411-5428, 2004.

[48] A. Menzel and P. Steinmann. On the continuum formulation of higher gradient plasticity for single and polycrystals. Journal of the Mechanics and Physics of Solids, 48(8):1777-1796, 2000.

[49] J.F. Nye. Some geometrical relations in dislocated crystals. Acta Metallurgica, 1(2):153-162, 1953.

[50] NJ Petch. The cleavage strength of polycrystals. Journal of the Iron and Steel Institute, 174:25-28, 1953.

[51] C. Polizzotto. Stress gradient versus strain gradient constitutive models within elasticity. International Journal of Solids and Structures, 51(9):1809-1818, 2014.

[52] B.D. Reddy. The role of dissipation and defect energy in variational formulations of problems in strain-gradient plasticity. Part 1: Polycrystalline plasticity. Continuum Mechanics and Thermodynamics, 23(6):527-549, 2011.

[53] B.D. Reddy. The role of dissipation and defect energy in variational formulations of problems in strain-gradient plasticity. Part 2: Single-crystal plasticity. Continuum Mechanics and Thermodynamics, 23(6):551-572, 2011.

[54] P. Steinmann. Views on multiplicative elastoplasticity and the continuum theory of dislocations. International Journal of Engineering Science, 34(15):1717-1735, 1996.

[55] P. Steinmann. Geometrical Foundations of Continuum Mechanics. Lecture Notes in Applied Mathematics and Mechanics. Springer, Berlin Heidelberg, 2015.

[56] J.S. Stölken and A.G. Evans. A microbend test method for measuring the plasticity length scale. Acta Materialia, 46(14):5109-5115, 1998.

[57] N. Taheri-Nassaj and H.M. Zbib. On dislocation pileups and stress-gradient dependent plastic flow. International Journal of Plasticity, 74:1-16, 2015. 
[58] M.A. Tschopp, D.E. Spearot, and D.L. McDowell. Atomistic simulations of homogeneous dislocation nucleation in single crystal copper. Modelling and Simulation in Materials Science and Engineering, 15(7):693, 2007.

[59] M.D. Uchic, D.M. Dimiduk, J.N. Florando, and W.D. Nix. Sample dimensions influence strength and crystal plasticity. Science, 305(5686):986-989, 2004.

[60] J.R. Willis. Bounds and self-consistent estimates for the overall properties of anisotropic composites. Journal of the Mechanics and Physics of Solids, 25(3):185-202, 1977.

[61] H.M. Zbib and E.C. Aifantis. A gradient-dependent model for the PortevinLe Chatelier effect. Scripta Metallurgica, 22(8):1331-1336, 1988.

[62] H.M. Zbib and E.C. Aifantis. A gradient-dependent flow theory of plasticity: Application to metal and soil instabilities. Applied Mechanics Reviews, 42(11):295-304, 1989.

[63] H.M. Zbib and E.C. Aifantis. On the gradient-dependent theory of plasticity and shear banding. Acta Mechanica, 92(1-4):209-225, 1992.

[64] H.M. Zbib, T.D. De la Rubia, and V. Bulatov. A multiscale model of plasticity based on discrete dislocation dynamics. Journal of Engineering Materials and Technology, Transactions of the ASME, 124(1):78-87, 2002.

[65] H.M. Zbib, T.D. De La Rubia, M. Rhee, and J. P. Hirth. 3d dislocation dynamics: Stress-strain behavior and hardening mechanisms in fcc and bcc metals. Journal of Nuclear Materials, 276(1):154-165, 2000.

[66] H.M. Zbib, M. Rhee, and J.P. Hirth. On plastic deformation and the dynamics of 3d dislocations. International Journal of Mechanical Sciences, 40(2-3):113-127, 1998. 


\section{Appendix}

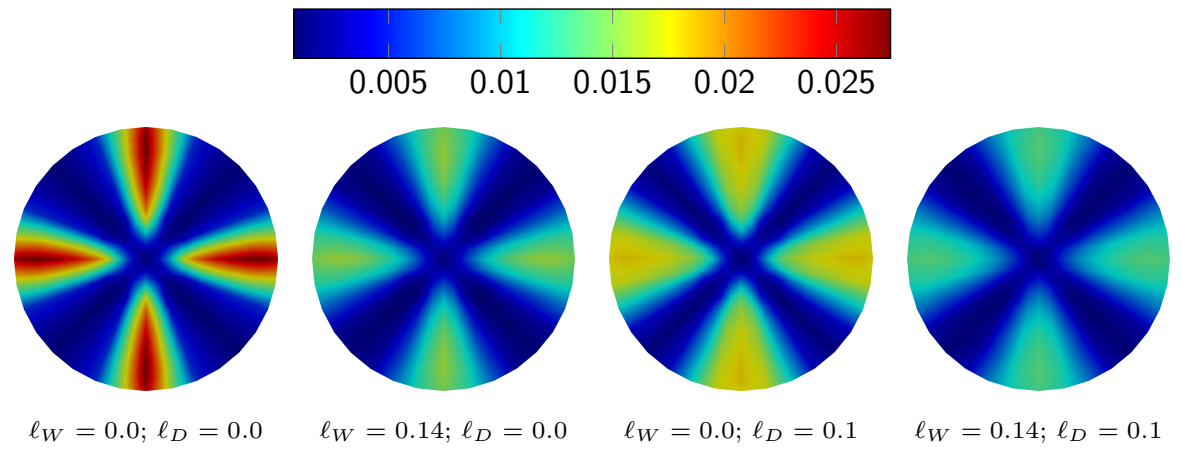

Figure 10: Norm of dislocation densities at twist of $5 \mathrm{~mm}^{-1}$.

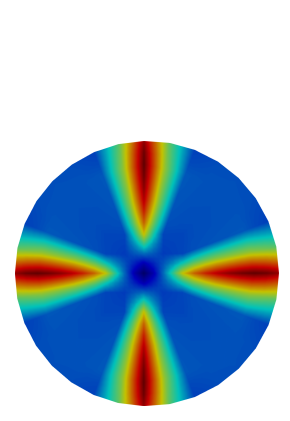

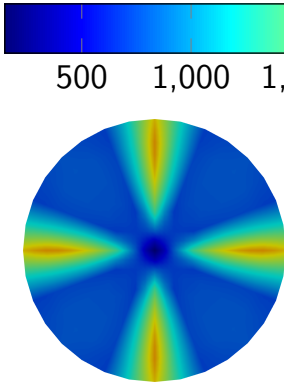

$\ell_{W}=0.14 ; \ell_{D}=0.0$

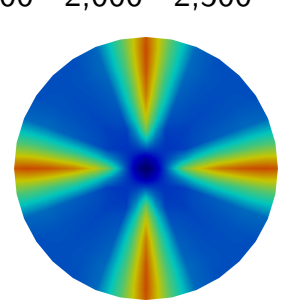

$\ell_{W}=0.0 ; \ell_{D}=0.1$

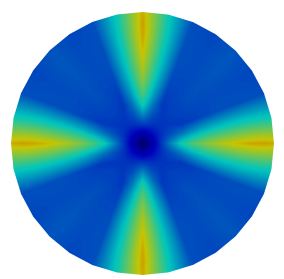

$\ell_{W}=0.14 ; \ell_{D}=0.1$

Figure 11: Norm of disequilibrium densities at twist of $5 \mathrm{~mm}^{-1}$.

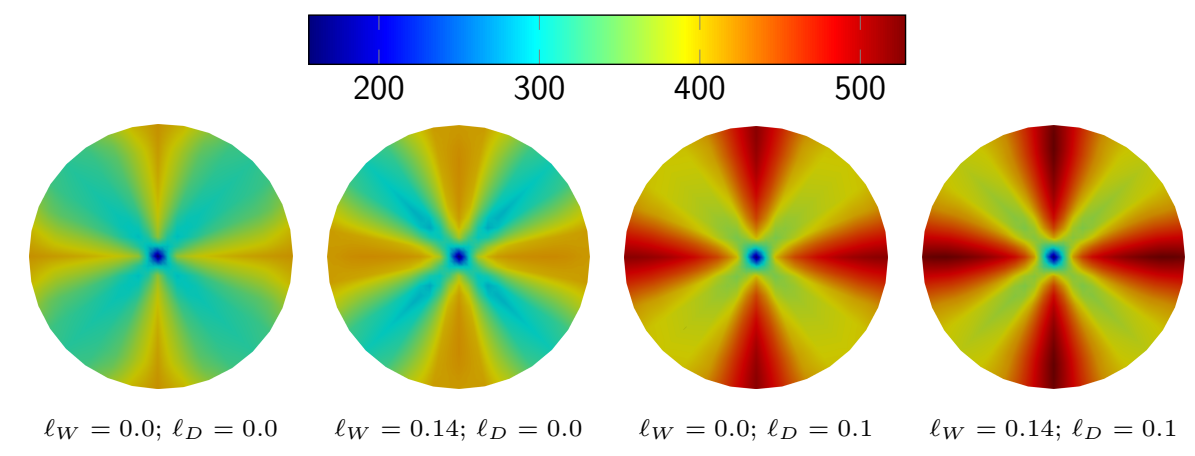

Figure 12: Von Mises stress at twist of $5 \mathrm{~mm}^{-1}$. 


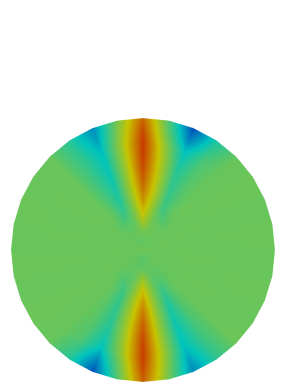

(a) $a=1$

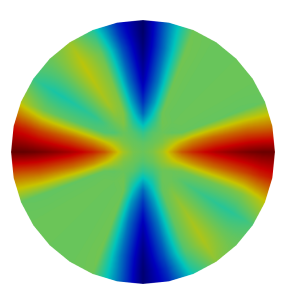

(e) $a=5$

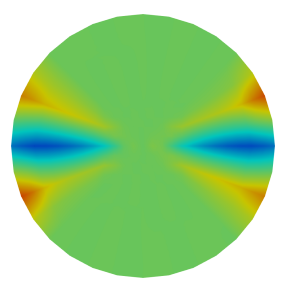

(i) $a=9$

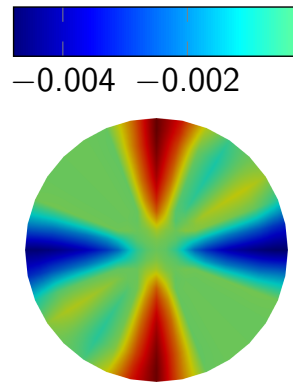

(b) $a=2$

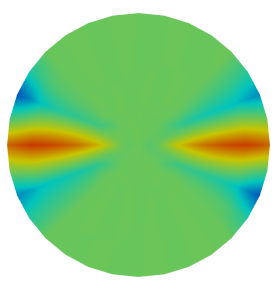

(f) $a=6$

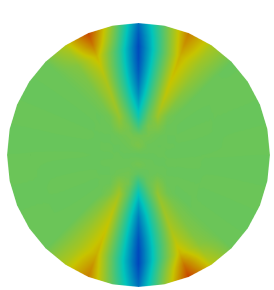

(j) $a=10$

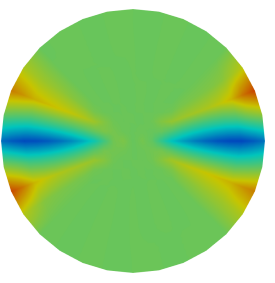

(c) $a=3$

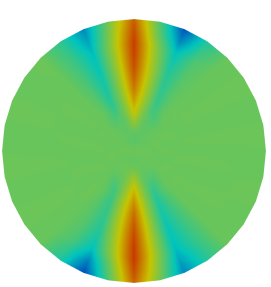

(g) $a=7$

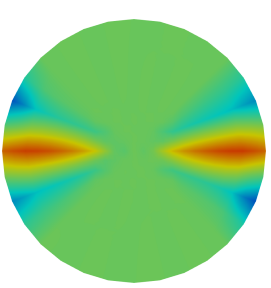

(k) $a=11$

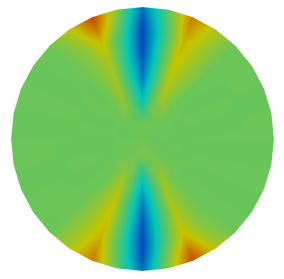

(d) $a=4$

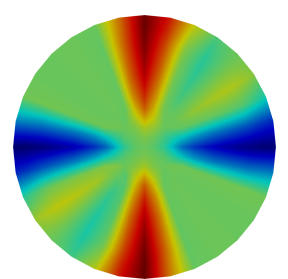

(h) $a=8$

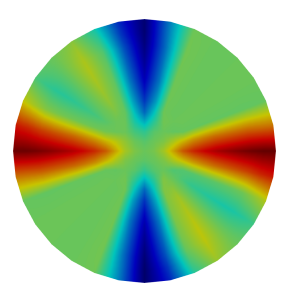

(l) $a=12$

Figure 13: Edge dislocation densities in $12 \mathrm{fcc}$ slip systems at twist of $5 \mathrm{~mm}^{-1}$. 


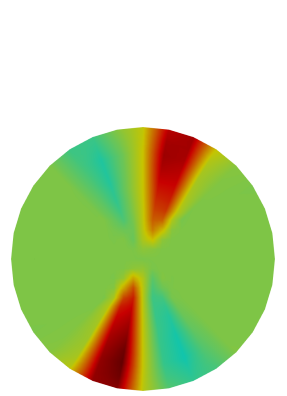

(a) $a=1$

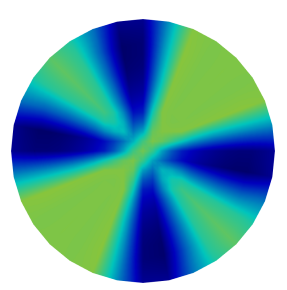

(e) $a=5$

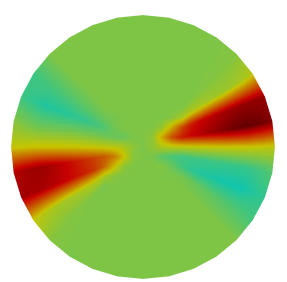

(i) $a=9$

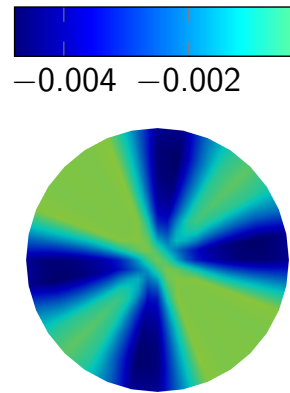

(b) $a=2$

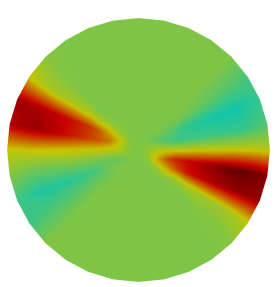

(f) $a=6$

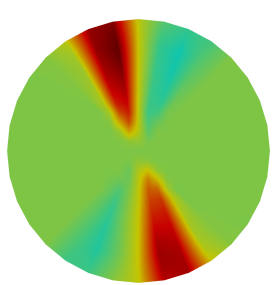

(j) $a=10$

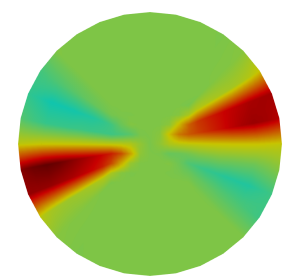

(c) $a=3$

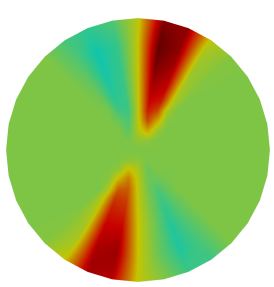

(g) $a=7$

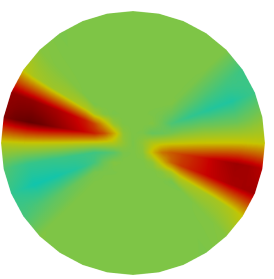

(k) $a=11$

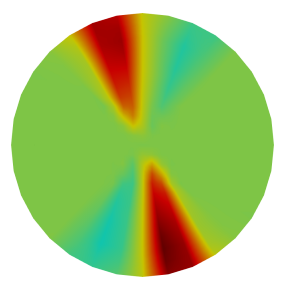

(d) $a=4$

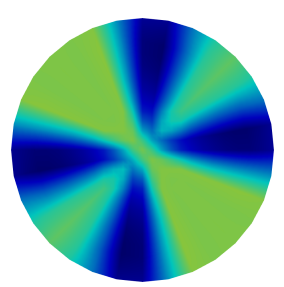

(h) $a=8$

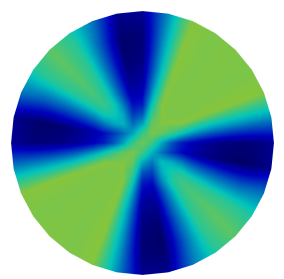

(l) $a=12$

Figure 14: Screw dislocation densities in $12 \mathrm{fcc}$ slip systems at twist of $5 \mathrm{~mm}^{-1}$. 


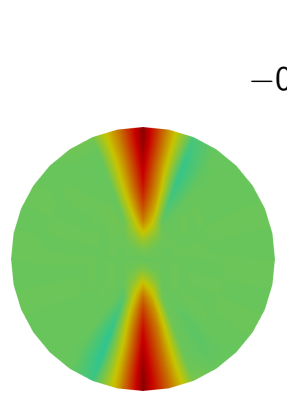

(a) $a=1$

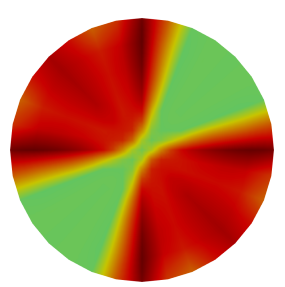

(e) $a=5$

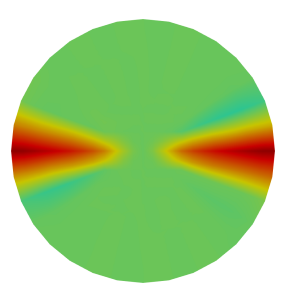

(i) $a=9$

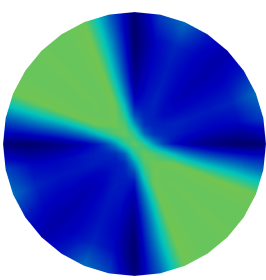

(b) $a=2$

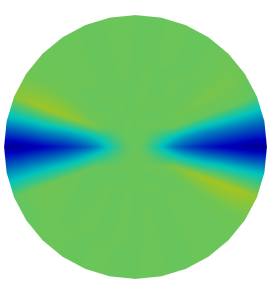

(f) $a=6$

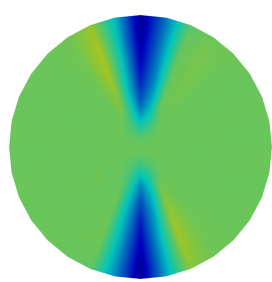

(j) $a=10$

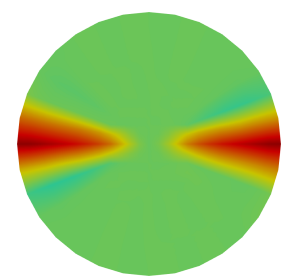

(c) $a=3$

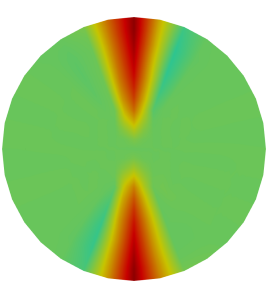

(g) $a=7$

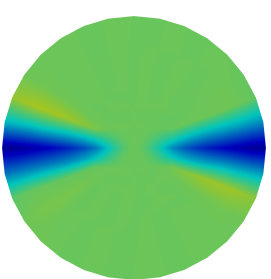

(k) $a=11$

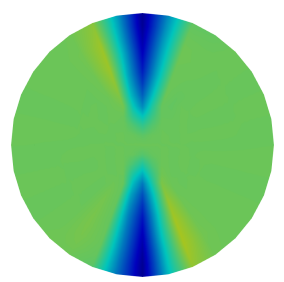

(d) $a=4$

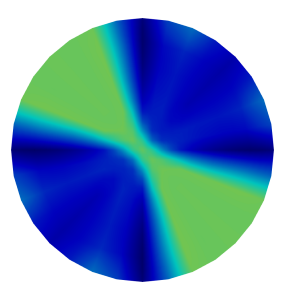

(h) $a=8$

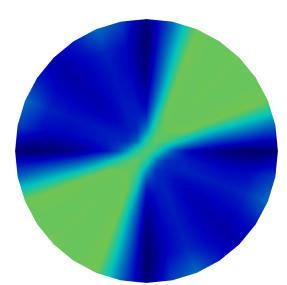

(l) $a=12$

Figure 15: Normal slip gradients in $12 \mathrm{fcc}$ slip systems at twist of $5 \mathrm{~mm}^{-1}$. 